\title{
Observations of shock waves in cloud cavitation
}

\author{
By G. E. REISMAN, Y.-C. WANG AND C. E. BRENNEN \\ California Institute of Technology, Pasadena, CA 91125, USA
}

(Received 20 November 1996 and in revised form 10 September 1997)

This paper describes an investigation of the dynamics and acoustics of cloud cavitation, the structures which are often formed by the periodic breakup and collapse of a sheet or vortex cavity. This form of cavitation frequently causes severe noise and damage, though the precise mechanism responsible for the enhancement of these adverse effects is not fully understood. In this paper, we investigate the large impulsive surface pressures generated by this type of cavitation and correlate these with the images from high-speed motion pictures. This reveals that several types of propagating structures (shock waves) are formed in a collapsing cloud and dictate the dynamics and acoustics of collapse. One type of shock wave structure is associated with the coherent collapse of a well-defined and separate cloud when it is convected into a region of higher pressure. This type of global structure causes the largest impulsive pressures and radiated noise. But two other types of structure, termed 'crescent-shaped regions' and 'leading-edge structures' occur during the less-coherent collapse of clouds. These local events are smaller and therefore produce less radiated noise but the interior pressure pulse magnitudes are almost as large as those produced by the global events.

The ubiquity and severity of these propagating shock wave structures provides a new perspective on the mechanisms reponsible for noise and damage in cavitating flows involving clouds of bubbles. It would appear that shock wave dynamics rather than the collapse dynamics of single bubbles determine the damage and noise in many cavitating flows.

\section{Introduction}

The most severe cavitation noise and erosion are produced when many bubbles collapse in close proximity to each other. This type of cavitation is commonly referred to as cloud cavitation. The very destructive effects which are observed to occur when such clouds form and collapse in a flow have been documented by, for example, Knapp (1955), Bark \& van Berlekom (1978) and Soyama, Kato \& Oba (1992). The generation of cavitation clouds may occur naturally as a result of the shedding of bubble-filled vortices, or it may be the response to a periodic disturbance imposed on the flow. Common examples of imposed fluctuations are the interaction between rotor and stator blades in a pump or turbine and the interaction between a ship's propeller and the non-uniform wake created by the hull.

Numerous investigators (Wade \& Acosta 1966; Bark \& van Berlekom 1978; Shen \& Peterson 1978, 1980; Blake, Wolpert \& Geib 1977; Bark 1985; Franc \& Michel 1988; Hart, Brennen \& Acosta 1990; Kubota et al. 1989; Kubota, Kato \& Yamaguchi 1992; Le, Franc \& Michel 1993; de Lange, de Bruin \& van Wijngaarden 1994) have studied the complicated flow patterns involved in the production and collapse of cloud cavitation on a hydrofoil. As early as 1964, Jakobsen suggested that a 
static bubbly condensation shock occurs in the bubbly closure region of a large attached cavity and provides the basic mechanism for cavity closure. The radiated noise produced by cloud cavitation is characterized by pressure pulses of very short duration and large magnitude. These pressure pulses were measured by Bark (1985), Bark \& van Berlekom (1978), Le et al. (1993), and Shen \& Peterson $(1978,1980)$. More recently, McKenney \& Brennen (1994) qualitatively related the acoustic signature of a cavitating cloud to the dynamics of the unsteady cavitation on an oscillating hydrofoil.

Analytical studies of the dynamics of cavitation clouds can be traced to the early work of van Wijngaarden (1964) who modelled the behaviour of a collapsing layer of bubbly fluid next to a solid wall. Later investigators explored numerical methods which treat the individual bubbles (Chahine 1982) and continuum models which, for example, address the behaviour of shock waves in a bubbly liquid (Noordzij \& van Wijngaarden 1974, Kameda \& Matsumoto 1995) and identified the natural frequencies of a spherical cloud of bubbles (d'Agostino \& Brennen 1983). Indeed the literature on the linearized dynamics of clouds of bubbles has grown rapidly (see, for example, Omta 1987; d'Agostino \& Brennen 1989; Prosperetti 1988). However, apart from the weakly nonlinear analyses of Kumar \& Brennen $(1991,1992,1993 a)$, only a few papers have addressed the highly nonlinear processes involved during the collapse of a cloud of bubbles. Chahine \& Duraiswami (1992) have conducted numerical simulations using a number of discrete bubbles and demonstrated how the bubbles on the periphery of the cloud develop inwardly directed re-entrant jets. However, most clouds contain many thousands of bubbles and it therefore is advantageous to examine the nonlinear behaviour of continuum models.

Another perspective on the subject of collapsing clouds was that introduced by Mørch (1980, 1981, 1982) and Hanson, Kedrinskii \& Mørch (1981). They suggested that the collapse of a cloud of bubbles involves the formation and inward propagation of a shock wave and that the geometric focusing of this shock at the centre of the cloud creates the enhancement of the noise and damage potential associated with cloud collapse. In the following section we summarize some of the results of numerical studies intended to investigate the dynamics and acoustics of a spherical cloud of bubbles.

The experiments described in subsequent sections were intended to shed light on the physical processes responsible for the dynamics and acoustics of real (nonspherical) clouds, specifically those occurring on a finite-span hydrofoil. We relate these observations to the other investigations mentioned above.

\section{Dynamics of a spherical cloud: numerical investigations}

Recently Wang \& Brennen (1994, 1995) (see also Wang 1996) have used the mixture models employed earlier by d'Agostino \& Brennen $(1983,1989)$ to study the nonlinear growth and collapse of a spherical cloud of bubbles. The purpose was to further the investigations of the mechanics of collapsing clouds begun by van Wijngaarden (1964) and, in particular, to study the phenomenon of shock wave formation described by Mørch and Kedrinskii and their co-workers (Mørch 1980, 1981, 1982; Hanson et al. 1981). They surmised that the collapse of a cloud of bubbles involves the formation and inward propagation of a shock wave and that the geometric focusing of this shock at the centre of the cloud creates the enhancement of the noise and damage potential associated with cloud collapse.

In Wang \& Brennen $(1994,1995)$ a finite cloud of nuclei is subjected to an episode of low pressure which causes the cloud to cavitate; the pressure then returns to the 


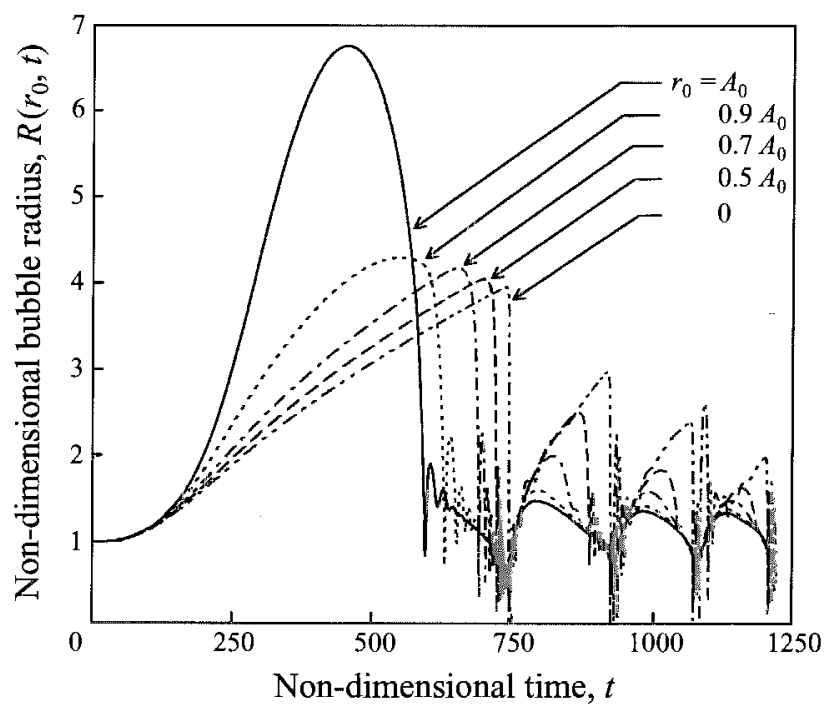

FIGURE 1 . The time history of the dimensionless bubble size at five different positions in the cloud for $\sigma=0.45, C_{r \min }=-0.75, \alpha_{0}=3 \%, A_{0}=100$, and $D / A_{0}=5$. The cloud interaction parameter, $\beta$, is approximately 300 in this case. The low-pressure episode extends from $t=0$ to $t=500$.

original level causing the cloud to collapse. The initial pressure level is characterized by a cavitation number, $\sigma$, and the low-pressure episode is characterized by a minimum pressure coefficient, $C_{p m i n}$, and a duration, $D / U$, where $D$ and $U$ are respectively the typical dimension and velocity of the cavitating flow. The initial radius and void fraction of the cloud are denoted by $A_{0}$ and $\alpha_{0}$ respectively and the initial radius of the bubbles within the cloud is denoted by $R_{0}$.

In carrying out numerical calculations of this characteristic cloud cavitation problem, Wang \& Brennen found that the 'cloud interaction' parameter, $\beta$, defined as

$$
\beta=\alpha_{0}\left(1-\alpha_{0}\right) A_{0}^{2} / R_{0}^{2},
$$

is crucially important for the dynamics and acoustics of the cloud. Earlier linear and weakly nonlinear studies of cloud dynamics (d'Agostino \& Brennen 1983, 1989; Kumar \& Brennen 1991, 1992, 1993a) showed that the cloud natural frequency is strongly dependent on this parameter. If $\beta$ is small, the natural frequency of the cloud is close to that of the individual bubbles in the cloud. In other words, the bubbles in the cloud tend to behave as individual units in an infinite fluid and the bubble/bubble interaction effects are minor. On the other hand, bubble interaction effects dominate when the value of $\beta$ is greater than order one. Then the collective oscillation of bubbles in the cloud results in a cloud natural frequency which is lower than the natural frequency of individual bubbles.

Some of the results obtained by Wang \& Brennen are shown in figures 1,2 and 3. Figures 1 and 3 present examples of the bubble-size time history for five different Lagrangian radial locations, $r_{0}$, within the cloud, from the surface, $r_{0}=A_{0}$, to the centre, $r_{0}=0$. Figure 1 is for a large value of $\beta$ in which the bubble interaction effects are strong. All the bubbles in the cloud grow almost in phase. However, because of the strong bubble/bubble interaction, bubble growth is severely constrained and the bubble growth rate within the cloud is much smaller than that near the surface. In other words, the bubbles in the interior are shielded by the outer shell of bubbles and 


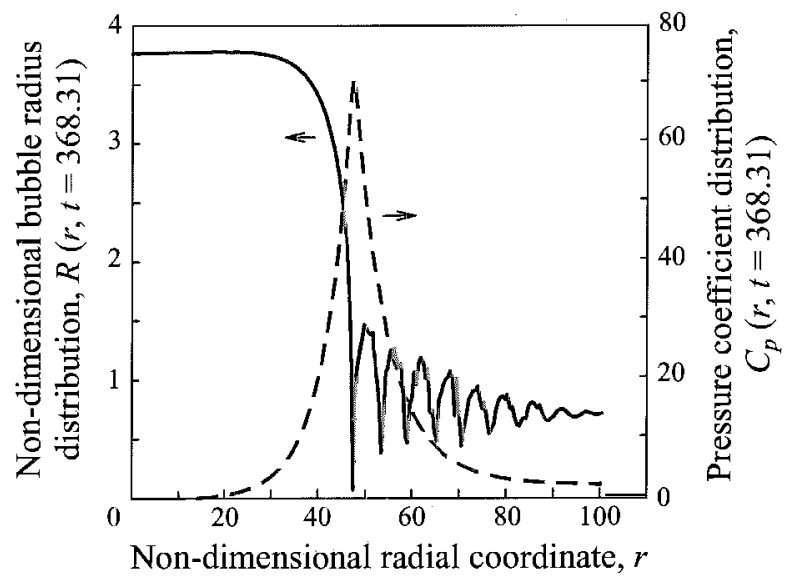

FIGURE 2. Bubble size and pressure distributions in the shock wave at a sample moment in time, $t=368.31$. Parameters are $\sigma=0.45, C_{\text {pmin }}=-0.75, \alpha_{0}=0.5 \%, A_{0}=100$, and $D / A_{0}=2.5$.

grow to a smailer maximum size. This shielding effect is typical of the bubble/bubble interaction phenomenon in cavitating cloud dynamics (d'Agostino \& Brennen 1983, 1989; Omta 1987; Smereka \& Banerjee 1988; Chahine \& Duraiswami 1992).

In the case of large $\beta$, as illustrated in figure 1 , the bubbles on the surface of the cloud collapse first and the collapse propagates inward creating a bubbly shock wave. Figure 2 shows the spatial distribution of bubble radius and pressure at one moment in time when the shock wave has progressed inward to a position about half the Lagrangian radius of the cloud. The structure of this shock is very similar to those in the bubbly flows investigated by Noordzij \& van Wijngaarden (1974) and other investigators (see, for example, Brennen 1995; Kameda \& Matsumoto 1995): the shock is composed of a series of rebounds and secondary collapses. The locations with small bubble size represent regions of low void fraction and higher pressure due to the local bubble collapse.

As the shock front passes bubbles and causes them to collapse, a very large pressure pulse is produced, as shown in figure 2. The shock wave strengthens considerably as it propagates into the cloud primarily because of geometric focusing. One consequence of this can be seen in figure 1; the closer the bubbles are to the cloud centre, the smaller the size to which they collapse. Very complicated bubble/bubble interactions are observed and very high pressures are generated when the focusing shock reaches the centre of the cloud. Then a spreading expansion wave causes all bubbles to grow and begins another cycle of cloud oscillation.

Very different dynamics occur when the cloud interaction parameter is small and a typical bubble time history under these conditions is shown in figure 3 which should be contrasted with figure 1 . Now, the bubbles grow more 'freely' to a large size. However, the bubbles close to cloud centre still grow more slowly than the bubbles near the surface and, consequently, the maximum size of the bubbles on the surface can be up to an order of magnitude larger. As a result, the central bubbles collapse first and form an outgoing shock which starts at the centre and weakens as it propagates outward. Therefore, there is no geometric focusing and the resulting noise produced is much smaller.

It is important to determine the acoustic consequences associated with the cloud dynamics described above. For this reason we examine the far-field acoustic noise 


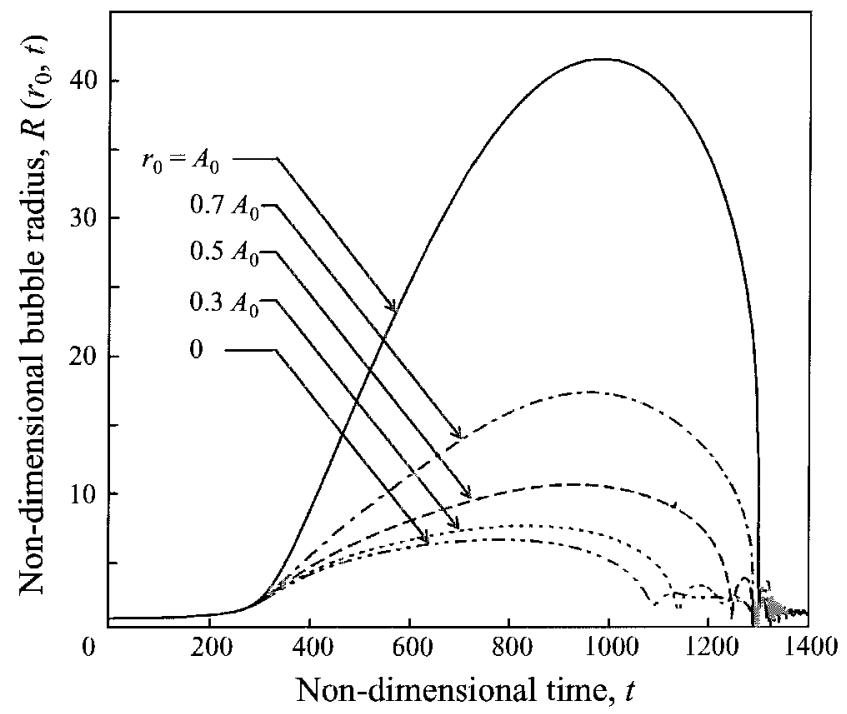

FIGURE 3. The time history of the dimensionless bubble size at five different positions in the cloud for $\alpha_{0}=0.03 \%$ and $D / A_{0}=10$. Other parameters as in figure 1 . The cloud interaction parameter, $\beta \approx 3$.

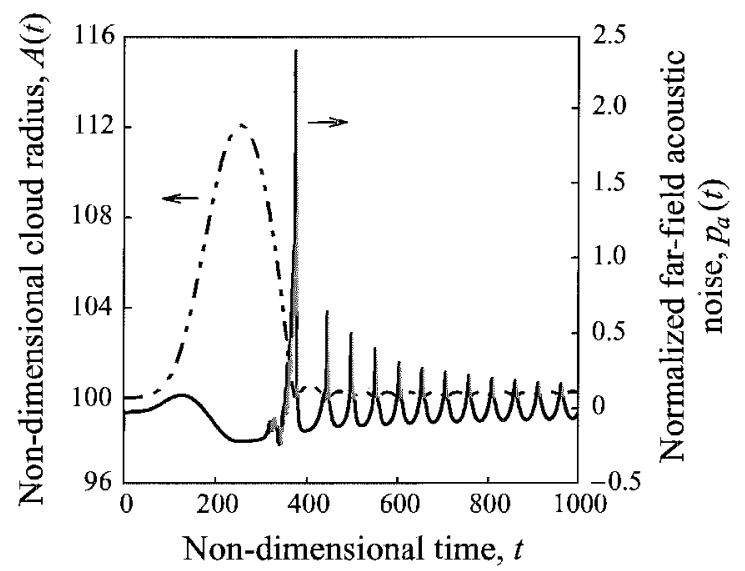

FIGURE 4. The time history of the dimensionless cloud radius and the resulting far-field acoustic noise (normalized by $\frac{1}{2} \rho U^{2} D / \mathscr{R}$ ) for the case of figure 2 .

produced by the volumetric acceleration of the cloud. If we denote the dimensional time-varying volume of the cloud by $V(t)$, it follows that the dimensional form of the time-varying far-field acoustic pressure is given by (Blake 1986)

$$
p_{a}(t)=\frac{\rho}{4 \pi \mathscr{R}} \frac{\mathrm{d}^{2} V(t)}{\mathrm{d} t^{2}},
$$

where $p_{a}$ is the radiated acoustic pressure, $\rho$ is the liquid density and $\mathscr{R}$ is the distance from the cloud centre to the point of measurement. The acoustic effects generated by individual bubbles are minor in the far field and will be neglected. Then the radiated noise is simply given by the volume of the cloud and a typical calculation of the cloud radius and the far-field noise is presented in figure 4 for a case in which $\beta \gg 1$. Note 
that, unlike a single bubble, the cloud only decreases to a size marginally smaller than its equilibrium size during the collapse process. However, the local void fraction within the cloud undergoes large changes. When the enhanced shock wave reaches the centre of the cloud, extremely high radiated noise is produced. Subsequent cloud collapses also produced radiated pulses. Normally, after several collapse and rebound cycles, the cloud will begin to oscillate at its natural frequency.

\section{Experiments}

The experiments were conducted in the low turbulence water tunnel (LTWT) at Caltech, a closed-circuit facility with test section dimensions of $30.5 \mathrm{~cm} \times 30.5 \mathrm{~cm} \times$ $2.5 \mathrm{~m}$ (Gates 1977). Two finite-span hydrofoils with a rectangular planform, a chord $c$ of $15.8 \mathrm{~cm}$ and a span of $17.8 \mathrm{~cm}$, were reflection-plane mounted in the floor of the test section as described in Hart et al. (1990) and shown in the sketch of figure 5. The thinner foil had a NACA 64A309 section; the other was specially chosen to allow the installation mounting of pressure transducers and had a modified NACA 0021 section with increased thickness between the mid-chord and the trailing edge. A $750 \mathrm{~W}$ DC motor is connected to a four-bar linkage which oscillates the foil in pitch about an axis near the centre of pressure, 0.38 of a chord from the leading edge. The mean angle of attack, the oscillation amplitude and frequency (up to $50 \mathrm{~Hz}$ ) are adjustable. An optical shaft encoder mounted on the DC motor provides a digital signal which was used to correlate the foil motion with the acoustic measurements and the high-speed movies.

The unsteady pressures generated by the cavitation on the hydrofoil were measured by several transducers. A PCB model HS113A21 piezo-electric pressure transducer (denoted by \#F) with a flat frequency response up to $100 \mathrm{kHz}$ was mounted flush with the floor of the test section, $5 \mathrm{~cm}$ downstream of the trailing edge of the foil. Another HS113A21 transducer (\#C) was mounted in the tunnel ceiling at approximately the same axial location. Four PCB model $105 B 02$ pressure transducers (flat frequency response to $50 \mathrm{kHz}$, face diameter about $3 \mathrm{~mm}$ ) were recess-mounted inside the foil at locations $26 \%$ span from the foil base and $30 \%, 50 \%, 70 \%$ and $90 \%$ chord from the leading edge. These surface transducers are respectively denoted by \#1 to \#4. The recess-mounting allowed the transducer axes to be placed along the span, thus minimizing the foil thickness. Parenthetically, we note that several earlier experiments with less robust flush-mounted transducers ended when the cavitation rapidly destroyed these devices (Reisman 1997).

By driving a transmitting hydrophone with a voltage impulse, the resonant frequencies of the transducer cavities were all determined to be about $35 \mathrm{kHz}$. These values are in good agreement with the Helmholtz resonant frequency of $38 \mathrm{kHz}$ calculated for the recess. Great care was taken to avoid the presence of air bubbles in the cavities and the impulse response testing was able to confirm their absence by detecting no change in the cavity resonant frequency. All data taken by the foil transducers were digitally low-pass filtered at $20 \mathrm{kHz}$ to avoid any effect of cavity resonance. The transmitting hydrophone was also used along with a second receiving hydrophone to confirm the manufacturer's calibration of the foil transducers (Reisman 1997). The output signals from all six transducers were low-pass filtered (to avoid aliasing) at $45 \mathrm{kHz}$ ( $70 \mathrm{KHz}$ for the NACA $64 \mathrm{~A} 309$ foil) and recorded digitally at a sampling rate of $100 \mathrm{kHz}(143 \mathrm{kHz}$ for the NACA $64 \mathrm{~A} 309$ foil).

A good measure of the magnitude of the cavitation pulses contained in the transducer output is the acoustic impulse, I (Ceccio \& Brennen 1991; Kuhn de Chizelle, 


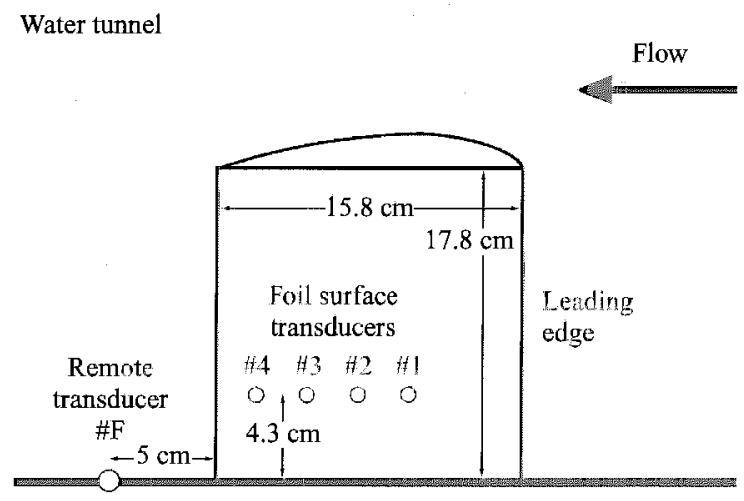

FiguRE 5. Sketch of the hydrofoil in the tunnel and the location of the pressure transducers.

Ceccio \& Brennen 1995). In the present analysis this defined as the area under the pulse,

$$
I=\int_{t_{1}}^{t_{2}} p_{a}(t) \mathrm{d} t
$$

where $t_{1}$ and $t_{2}$ are the times for which $p_{a}$ exceeds a threshold pressure, $p_{t}$. A threshold of $20 \mathrm{kPa}$ was used for the floor and ceiling transducers while $200 \mathrm{kPa}$ was used for the foil surface transducers. The determination of the optimal threshold is a complicated process which is discussed elsewhere (Reisman 1997). The data presented here were obtained from 40 samples at each operating condition where a single sample is defined either as one oscillation cycle or as a $0.066 \mathrm{~s}$ time period during the stationary foil experiments. For presentation purposes, non-dimensional acoustic impulses, $I^{*}$, were calculated by dividing $I$ by $\rho U c^{2} / 16 \pi \mathscr{R}$ where $U$ is the tunnel velocity and $\mathscr{R}$ is the distance from the cavitation collapse to transducer \#F.

High-speed movies with a framing rate of 500 f.p.s. were taken to assist in the interpretation of the pressure transducer output. These movies were synchronized with the pressure signals by triggering a timing light simultaneously with commencement of data acquisition.

\section{Acoustic measurements}

Preliminary studies of the dynamics and acoustics of cloud cavitation were performed using the NACA 64A309 hydrofoil and the transducer mounted in the tunnel floor $(\# \mathrm{~F})$. Figure 6 illustrates typical cloud and sheet cavitation structures present on the suction surface of the foil during these experiments (see also Hart et al. 1990; McKenney \& Brennen 1994; Reisman, McKenney \& Brennen 1994, Reisman 1997). In the photograph, tip-vortex cavitation is visible at the upper left and sheet and cloud cavitation are both present. The dynamics of these cavitation processes will be described in detail later.

In this section we briefly surnmarize the results of these preliminary experiments (Reisman et al. 1994). They were conducted with a mean angle of attack, $\overline{\alpha_{f}}$, of $9^{\circ}$, an oscillation amplitude, $\Delta \alpha_{f}$, of $\pm 5^{\circ}$, a range of reduced frequencies, $k$ (the radian frequency divided by $2 U / c$ ), from 0.55 to 0.93 , a range of cavitation numbers, $\sigma$, from 


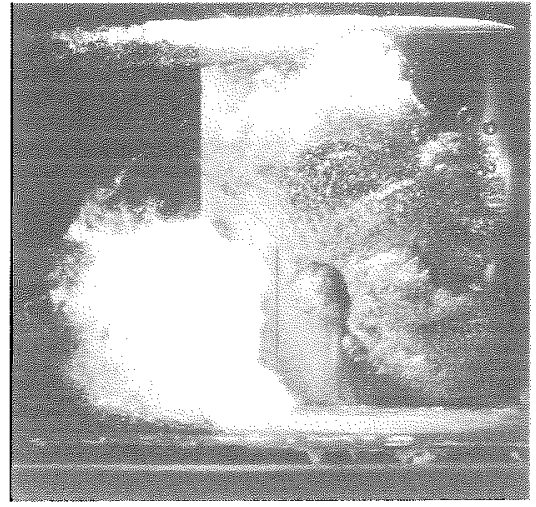

FIGURE 6. Cloud cavitation on the oscillating NACA 64A309 hydrofoil at $\sigma=1.2, k=0.9$, $U=8 \mathrm{~m} \mathrm{~s}^{-1}$. View of the suction surface with the flow from right to left, the base at the bottom and the tip and tip vortex at the top.

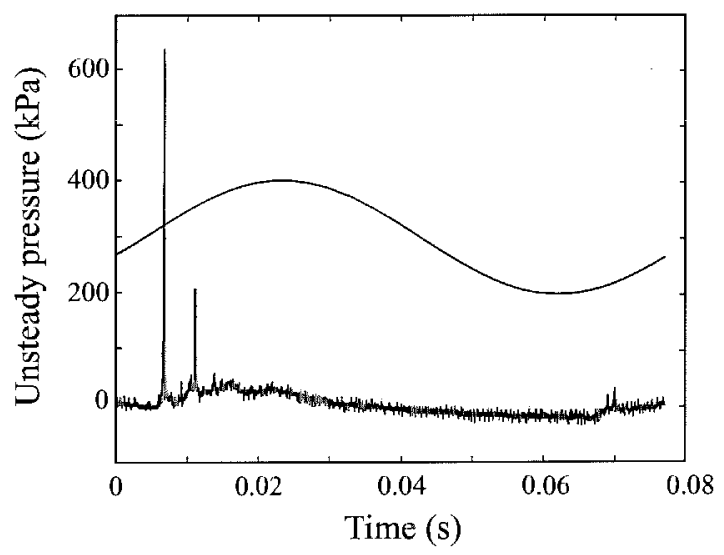

FIGURE 7. Typical output from transducer \#F located in the tunnel test section floor. The sinusoid qualitatively represents the instantaneous angle of attack of the NACA 64A309 foil. Data for $\sigma=1.0, k=0.8, U=8 \mathrm{~m} \mathrm{~s}^{-1}$ and an air content of 4-5 p.p.m.

0.9 to 1.5 and at a tunnel velocity, $U$, of $8 \mathrm{~m} \mathrm{~s}^{-1}$. The total air content was measured before and after each run. For most of the experiments, the air content was in the range of 8-10 p.p.m. However, some experiments were repeated with 4-5 p.p.m. Data for approximately 40 cycles were obtained at each condition.

Figure 7 presents a typical unsteady pressure signal from the floor transducer (before high-pass filtering) during one cycle, along with a curve indicating the instantaneous angle of attack. The origin of the time axis corresponds to $\alpha=7.9^{\circ}$ with $\alpha$ increasing. Two clear pulses at about $\alpha=10^{\circ}$ represent the substantial acoustic radiation by the cloud during this particular cycle. The larger pulse has a peak value of $650 \mathrm{kPa}$ and was one of the largest recorded by the floor transducer; most pulses were of the order of a few bars in magnitude. The multiple peaks seen in this signal are characteristic of many of the signals recorded during these experiments. Multiple peaks could result from overlapping events, from a series of collapses and rebounds or from multiple shock waves propagating through the cloud as discussed later in $\S 14$. 


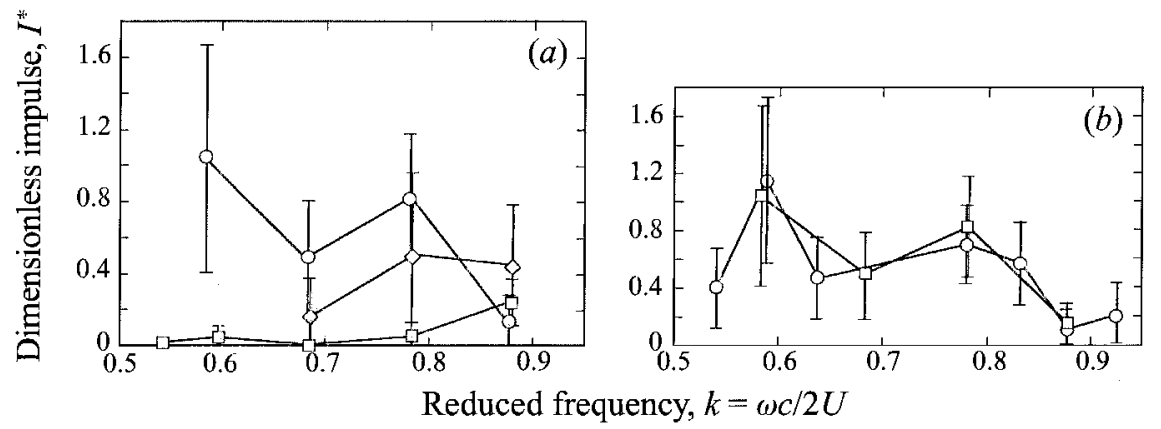

FIGURE 8. (a) Effect of cavitation number, $\sigma$, on the dimensionless impulse, $I^{*}$. Data shown for the NACA 64A309 foil with $\sigma=0.9(\diamond), \sigma=1.2(\odot), \sigma=1.5(\square)$ with $U=8 \mathrm{~m} \mathrm{~s}^{-1}$ and an air content of 7-10 p.p.m. (b) Effect of air content on dimensionless impulse, $I^{*}$. Data shown for 4-5 p.p.m.

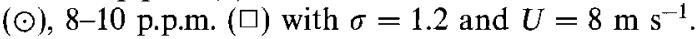

\section{Acoustic impulse}

The experimental results depicted in figure 8 illustrate the variation of the dimensionless impulse, $I^{*}$, with cavitation number, reduced frequency and air content. Each data point represents the average of all the impulses occurring during approximately 40 foil oscillation cycles. Within the parameter space, significant cycle-to-cycle variation was observed in both the physical attributes of the cavitation and the resulting impulse. A measure of this scatter is shown in figure 8 by vertical bars which represent one standard deviation above and below the average impulse value. The standard deviation ranges from approximately $60 \%$ of the mean for impulses greater than 0.4 to $120 \%$ of the mean for $I^{*}$ less than 0.4 . However it is important to emphasize that the repeatability of the mean value was approximately \pm 0.1 .

Figure $8(a)$ shows the change in impulse with reduced frequency, $k$, for different cavitation numbers. As expected, the cavitation number, $\sigma$, and reduced frequency, $k$, have a significant effect on the measured impulse. The highest cavitation number, $\sigma=1.5$, resulted in the lowest impulse (with one exception). With the same exception, the sound level produced at $\sigma=1.2$ exceeded the level measured at $\sigma=0.9$. In general, the noise appears to peak at some intermediate value of $\sigma$.

Arndt, Ellis \& Paul (1993), using a stationary hydrofoil, noted a similar nonmonotonic relationship between the noise level and $\sigma$, but this behaviour differs from that found by McKenney \& Brennen (1994). A possible explanation may be the fact that the present mean angle of attack, $\overline{\alpha_{f}}$, is larger than that used by McKenney \& Brennen (1994). This seems to alter the cavitation number at which the noise peaks. In the present experiments, it could be visually observed that, for $\sigma=0.9$, the sheet cavity not only covered nearly the entire surface of the hydrofoil, but also extended past the trailing edge for a large part of the oscillation cycle. At the lower $\overline{\alpha_{f}}$ used by McKenney \& Brennen (1994), however, the sheet cavity seldom covered more than about $60 \%$ of the foil surface.

Although the air content varied from 4 p.p.m. to 10 p.p.m. during the experiments, it appeared to have little effect on the noise, as shown in figure $8(b)$. This result was unexpected since, as shown by Reisman (1997), the injection of air into the sheet cavity, even at very low flow rates, results in a profound reduction in the measured acoustic impulse. A larger air content was therefore expected to produce a similar effect due to diffusion of air into the sheet cavity. The lack of any measurable effect of the air content can be attributed to the lack of significant diffusion during the relatively short 


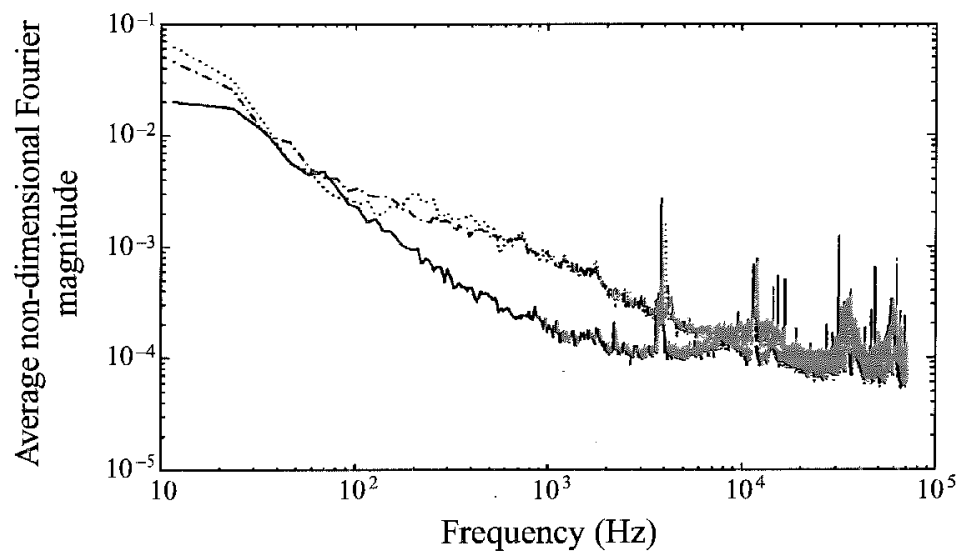

FIGURE 9. Comparison of the normalized spectra of unsteady pressures from the NACA 64A309 foil at three different cavitation numbers: $\sigma=1.5$ (solid line), $\sigma=1.2$ (dotted line), and $\sigma=0.9$ (dash-dot line). Data for $k=0.7, U=8 \mathrm{~m} \mathrm{~s}^{-1}$ and an air content of 7-10 p.p.m.

time period associated with the unsteady cavitation growth and collapse. This result is not unprecedented since Bark (1985) noted that the air content had no influence on cavitation structures observed in his oscillating hydrofoil experiments.

\section{Spectral analysis}

Further insight into cloud cavitation noise generation can be obtained by Fourier analysis of the radiated acoustic pressure. Figure 9 presents typical examples of cloud cavitation noise spectra. Each line in the figure corresponds to a single set of parameters and represents an average of approximately 40 Fourier transforms normalized using the method of Kumar \& Brennen (1993b). The spectra obtained from the current experiments exhibit a characteristic behaviour proportional to $f^{-n}$ over the frequency range $80-6000 \mathrm{~Hz}$, where $0.7>n>0.8$. This compares reasonably well with the roughly $f^{-1}$ behaviour of previous experimental results for cavitation noise spectra (see Fitzpatrick \& Strasberg 1956, Blake 1986, Brennen 1995) including the work of Blake et al. (1977) and Arakeri \& Shanmuganathan (1985).

Figure 9 presents the average spectrum for each of three cavitation numbers. It is clear that there is a significant change in the spectrum with cavitation number particularly in the range $100 \mathrm{~Hz}$ to $7 \mathrm{kHz}$. Moreover, the relative changes in magnitude in this range parallel the previously described changes in the acoustic impulse with $\sigma$. This result is similar to that obtained by Arndt et al. (1993) in their investigations of a stationary foil; they measured a non-monotonic relationship between the noise spectral density and $\sigma$ in the frequency range of $1 \mathrm{kHz}$ to $10 \mathrm{kHz}$. However, it differs from the monotonic increase in spectral density with decreasing cavitation number reported by Blake et al. (1977) for both sheet and travelling bubble cavitation on a stationary foil. Average spectra were also obtained for a series of reduced frequencies and for different air content, but showed little variation with either of these parameters (Reisman 1997).

The large peak at $4 \mathrm{kHz}$ appears in all spectra for the entire parameter space. It is even observed in the spectral analysis of data taken at high pressures when no cavitation was present on the foil. Since the the fundamental resonant frequency of 


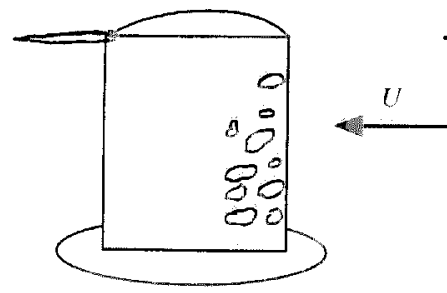

1. Travelling bubble cavitation

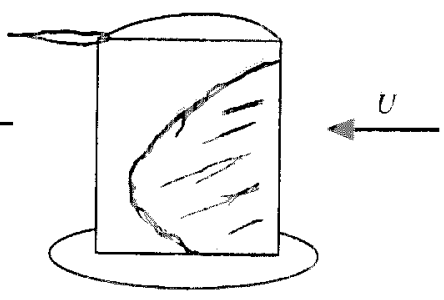

2. Maximum cavity length

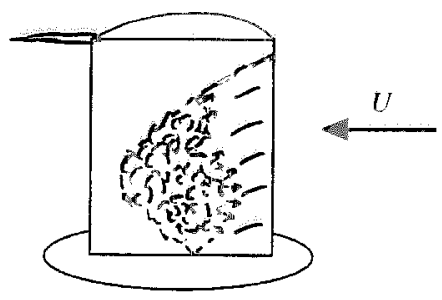

3. Re-entrant jet

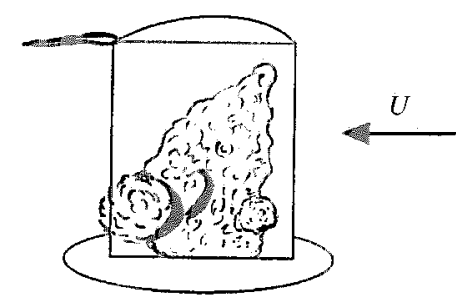

5. Local pulse phenomena

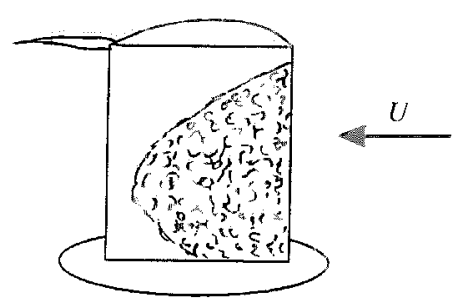

4. Bubbly mixture

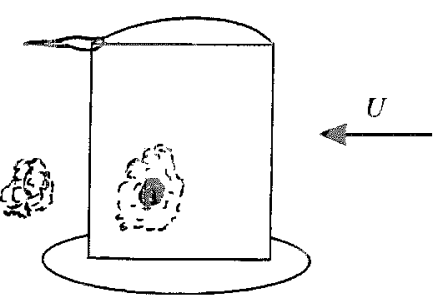

6. Global pulse phenomena

FIGURE 10. Cavitation patterns observed during a single oscillation cycle for a mean angle of attack of $5^{\circ}$. The angle of attack increases from sketch 1 to a maximum at sketch 2 and then decreases to a minimum at sketch 5 .

the LTWT test section is $3.5 \mathrm{kHz}$ (Reisman 1997) it seems likely that the $4 \mathrm{kHz}$ peak is an artifact of the LTWT facility.

\section{Stages of the cavitation cycle}

Detailed descriptions of cavity growth, collapse and cloud formation on hydrofoils have been given by many authors including Knapp (1955), Wade \& Acosta (1966), Bark (1985), McKenney \& Brennen (1994), Shen \& Peterson (1978, 1980), Blake et al. (1977), Franc \& Michel (1988), Hart et al. (1990), Kubota et al. (1989, 1992), Le et al. (1993), de Lange et al. (1994) and Kawanami et al. (1996). By viewing the high-speed motion pictures taken during the current experiments, a series of stages in the cloud cavitation process were identified. These stages are depicted schematically in figure 10 for a single foil oscillation cycle. In addition, selected frames from a high-speed movie of a single foil oscillation cycle are shown in figure 11. It should be noted that the description below and the sketches of figure 10 are based on a mean angle of attack of $5^{\circ}$. The high-speed motion picture depicted in figure 11 was taken 
G. E. Reisman, Y.-C. Wang and C. E. Brennen

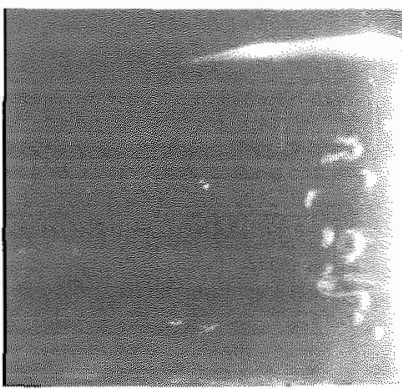

(a)

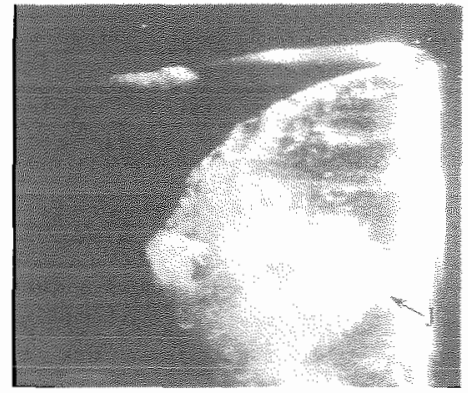

(c)

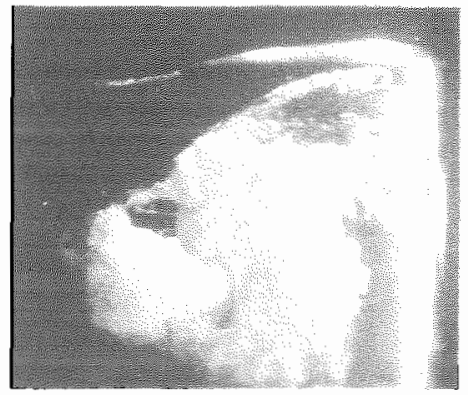

(e)

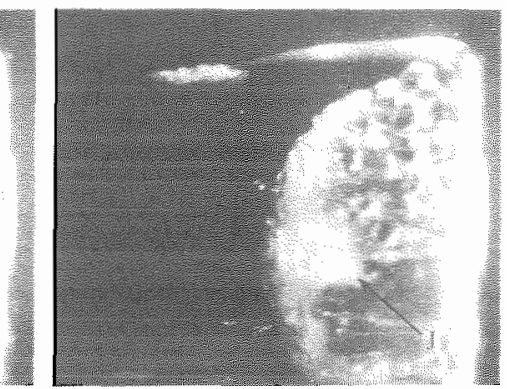

(b)

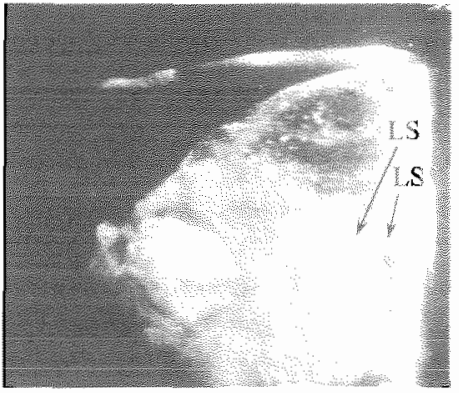

(d)

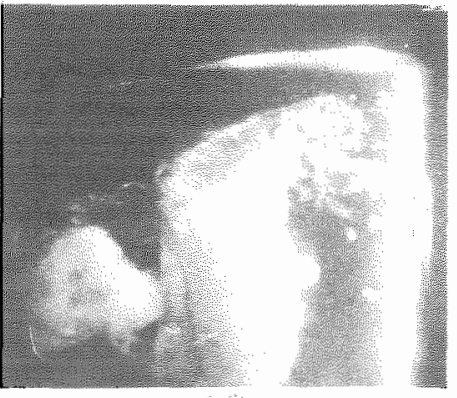

(f)

FIgURE 11. Cavitation during a single oscillation cycle as seen in selected frames from a high-speed motion picture of a single oscillation cycle of the NACA 0021 foil at $k=0.7, \sigma=0.95, U=8.5 \mathrm{~m} \mathrm{~s}^{-1}$, and $\overline{\alpha_{f}}=7^{\circ}$. View of the suction surface with the flow from the right to the left and the leading edge just inside the right-hand border. The front of the re-entrant jet is indicated $(J)$ in frames $(b)$ and $(c)$ and several local pulse structures are indicated (LS) in frame $(d)$.

with the foil oscillating at a mean angle of attack of $7^{\circ}$. Although the same structures are observed in the same order during experiments at both mean angles of attack, the instantaneous angle of attack at which each phenomenon occurred were different for the two cases.

During that part of the oscillation cycle when the instantaneous angle of attack, $\alpha_{f}$, is increasing, cavitation inception occurs in the tip vortex and is soon followed by travelling bubble cavitation in the area of $C_{\text {pmin }}$ as can be seen in frame $(a)$ of figure 11. The travelling bubble cavitation was observed to persist for a longer period of time on the thicker NACA 0021 foil than on the NACA 64A309 foil. As the angle of attack increases further, the bubbles coalesce into a single attached cavitation sheet which attains its maximum length as the angle of attack reaches a maximum. This process is depicted in frames $(b)$ and $(c)$ of figure 11 . Near the end of this process, a 
re-entrant liquid jet penetrates the attached cavity from downstream and flows toward the leading edge of the foil. This re-entrant jet mechanism was described by Knapp (1955) and further elucidated in the work of Wade \& Acosta (1966), Lush \& Skipp (1986), Kubota et al. (1992), de Lange et al. (1994) and Kawanami et al. (1996). The re-entrant jet does not, however, progress uniformly toward the leading edge. As can be seen in figure $11(b, c)$, the jet penetration is maximium at approximately $30 \%$ span (from the base of the foil). The arrow in these frames indicates the front or leading edge of the re-entrant jet.

The current observations indicate, however, that the processes which occur after the passage of the re-entrant jet are critical. The large pressure pulses on the foil surface, which will be described in greater detail below, were detected only after the re-entrant jet had passed the measurement location. The jet proceeds to the leading edge of the foil and, in the process, breaks up the attached sheet cavity into a bubbly mixture which detaches from the foil surface and accelerates downstream. The thickness of the bubbly mixture increases in the region through which the jet passes by mixing of the vapour/gas contained in the cavity with the surrounding liquid to create a larger volume of bubbly liquid. The breakup of the sheet cavity is seen in figure $11(d-f)$; the detachment of the bubbly mixture from the foil surface begins in frame $(d)$. Finally, the remains of the sheet cavity form a cloud of bubbles that undergoes a coherent collapse as it is convected into a region of higher pressure near the foil trailing edge. This collapse is shown in figure 16. Note that this collapse results in only a slight change in the cloud radius but a large change in the void fraction magnitude and distribution inside the cloud.

It should be ernphasized that these cloud cavitation structures are three-dimensional. Figure 12 contains sketches traced from high-speed motion pictures of both the profile and planform outlines of the cavitation and their evolution during one cycle of oscillation. The profile and planform movies were taken at the same operating conditions, but were not filmed simultaneously. Cycle-to-cycle variations in the details of the cloud cavitation are significant, but the overall pattern is quite repeatable. Sketch $(a)$ of figure 12 corresponds to a moment in the cycle at which $\alpha_{f}=10^{\circ}$ and is decreasing. The re-entrant jet has begun to penetrate the sheet cavity and its front is closest to the foil leading edge at a spanwise location about $30 \%$ from the base of the foil. The thickness of the attached shect cavitationi is of the order of the thickness of the foil. The region near the trailing edge was obstructed during the filming of the profile view, but the position of the re-entrant jet is apparent from the appearance of a bubbly mixture extending out beyond the profile of the sheet cavity. Sketches $(b),(c)$ and $(d)\left(\alpha_{f}=8.4^{\circ}, 6.9^{\circ}\right.$ and $5.4^{\circ}$, respectively) show the re-entrant jet moving toward the foil leading edge; as it does so, the bubbly mixture expands, reaching a stand-off distance from the foil of almost a half a chord. At first however, this expansion only occurs near the $30 \%$ span location. In figure $12(a-e)$ attached sheet cavitation is still present at other spanwise locations. In $(f)\left(\alpha_{f}=3.2^{\circ}\right.$ and increasing), the breakup of the attached sheet cavitation into a bubbly mixture is complete. The mixture continues to expand as it is convected downstream and the individual bubbles of the mixture collapse (sketches $g$ and $h$ with $\alpha_{f}=4.7^{\circ}$ and $7.7^{\circ}$ ). Travelling bubble cavitation reappears briefly in sketch $(g)$ and is observed in both the profile and planform views.

These profile and planform images are not unlike the descriptions of cloud cavitation in the two-dimensional hydrofoil experiments of de Lange et al. (1994), Shen \& Petersen (1978), Franc \& Michel (1988) and Kawanami et al. (1996). The most important difference in the present case is the substantial spanwise variation in the 

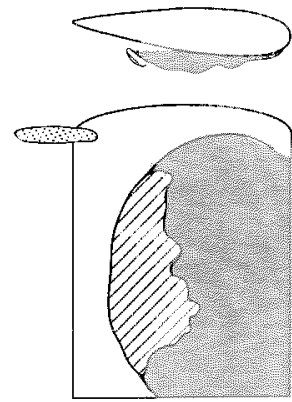

(a)

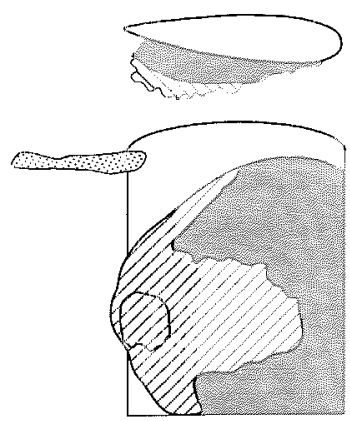

(c)

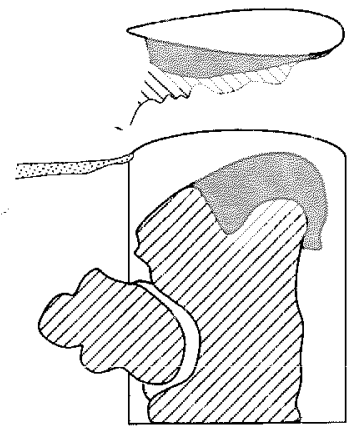

(e)
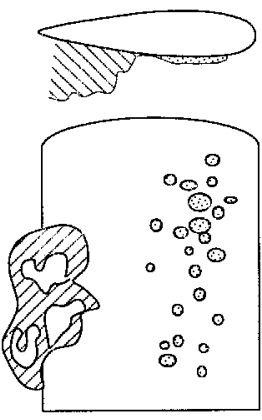

(g)

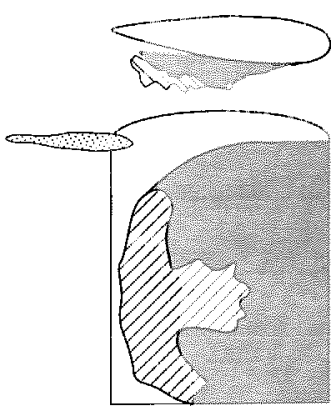

(b)

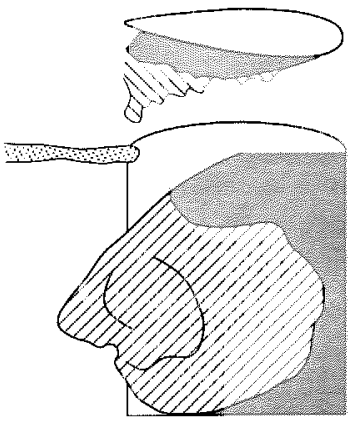

(d)

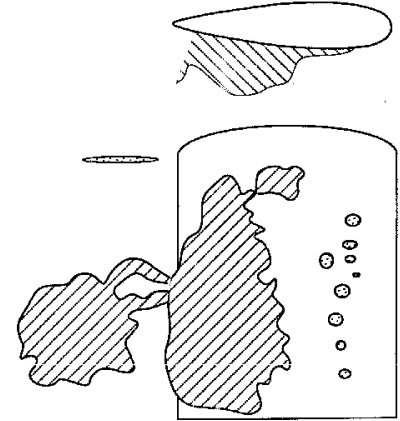

(f)
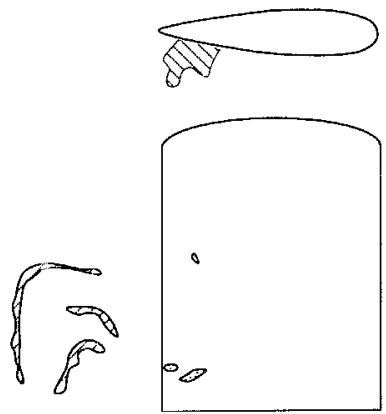

(h)

FIGURE 12. Profile and planform sketches of the cloud cavitation as traced from high-speed motion pictures of a single foil oscillation cycle. Shaded areas: sheet cavitation; hatched areas: bubbly mixture; dotted areas: travelling bubble or tip vortex cavitation. NACA 0021 foil oscillating at $k=0.7, \sigma=0.9-0.95, U=8.5 \mathrm{~m} \mathrm{~s}^{-1}, \overline{\alpha_{f}}=7^{\circ}$. 


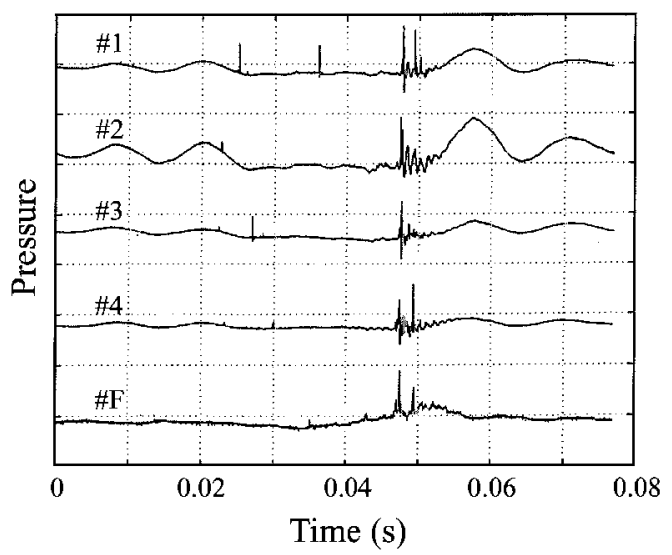

FIGURE 13. Typical signals from transducers \#1-\#4 and \#F during a single oscillation cycle of the NACA 0021 foil. The vertical scale is $1 \mathrm{MPa} /$ div. for the foil surface transducers, \#1-\#4, and $100 \mathrm{kPa} / \mathrm{div}$. for the floor transducer, \#F. Data for $\sigma=0.95, k=0.78, U=8 \mathrm{~m} \mathrm{~s}^{-1}$ and $\overline{\alpha_{f}}=5^{\circ}$.

cavitation and its evolution caused by the finite-span configuration of the hydrofoil. This leads to important three-dimensional variations in the thickness and void fraction of the cavitation.

\section{Pressure pulses due to cloud cavitation}

The high-speed motion pictures taken of cloud cavitation on the NACA 64A309 foil showed the presence of several interesting structures within the cavitation. To investigate these phenomena further, the NACA 0021 hydrofoil was fabricated in order to obtain measurements of the unsteady surface pressures which could then be correlated with the photographic record of the cavity and its structures. These experiments with the NACA 0021 foil were conducted at a mean angle of attack of $5^{\circ}$, at an oscillation amplitude of $\pm 5^{\circ}$ (except, of course for the stationary foil experiments), at cavitation numbers of $0.85,0.95$, and 1.05 , at tunnel velocities of $7.5 \mathrm{~m} \mathrm{~s}^{-1}, 8 \mathrm{~m} \mathrm{~s}^{-1}$ and $8.5 \mathrm{~m} \mathrm{~s}^{-1}$ and at reduced froquicncics of $0,0.76,1.00$ and 1.22 . The total air content was 9.3 p.p.m. for the oscillating foil experiments and 8.2 p.p.m. for the stationary foil experiments.

A typical set of output signals from the transducers is shown in figure 13 which represents a single foil oscillation cycle with the origin corresponding to the maximum angle of attack. As exemplified by figure 13, the output signals are characterized by very large-amplitude pressure pulses with magnitudes of the order of tens of atmospheres and typical durations of the order of tenths of milliseconds. As previously described in $\S 5$, the radiated acoustic pressure also contains pressure pulses and these are exemplified by the bottom trace in figure 13. The magnitude of the pulses measured by the transducer in the tunnel floor $(\# F)$ is of the order of one atmosphere. The closest approximation to the far-field pressure is that measured by the transducer in the ceiling of the tunnel test section (\#C); this also contains impulses, but these were less than $10 \mathrm{kPa}$ in amplitude. Although these far-field measurements are affected by both the directional dependence of the ceiling transducer and the tunnel test section acoustics, it is clear that there are order of magnitude differences in the amplitudes of the pulses measured on the foil, near the cavitating region and in the far field, respectively. 
In figure 13 the low-frequency variation present in the signal prior to the $0.025 \mathrm{~s}$ mark and after the $0.05 \mathrm{~s}$ mark was also observed at high cavitation numbers when the foil was not cavitating. This part of the signal is the result of stresses in the foil which are communicated through the transducer casings. This explanation is supported by the virtually instantaneous propagation of the low-frequency disturbance and by the observation of similar signals even when the foil was oscillating in air.

Pressure pulses radiated from and within cavitating flows have been measured before. Bark \& van Berlekom (1978) and Bark (1985) measured pulses of the order of tens of $\mathrm{kPa}$ at locations near a foil with cloud cavitation. These pulses are appropriately compared to the far-field measurements made with transducer \#C whose magnitudes are similar. They are significantly smaller than the pulses measured by transducers \#1-\#4 and \#F. This difference in amplitude could also be due to the significantly smaller velocities of the Bark \& van Berlekom experiments which were performed at $5 \mathrm{~m} \mathrm{~s}^{-1}$. Shen $\&$ Peterson (1978) recorded foil surface pulses comparable with the dynamic pressure (about $110 \mathrm{kPa}$ ) and therefore much smaller than measured in the present experiments. There are at least two explanations for this discrepancy. The primary reason is that the transducers used by Shen \& Peterson had a flat response only up to $2 \mathrm{kHz}$ whereas the present measurements exhibit substantial content above that frequency. In addition, both the amplitude of oscillation (up to $\left.1.55^{\circ}\right)$ and the mean angle of attack $\left(3.25^{\circ}\right)$ used by Shen \& Peterson were smaller than in the present experiments. More recently, Le et al. (1993) measured pulse magnitudes of up to $7 \mathrm{MPa}$ on a stationary foil at $\sigma=0.81, U=12 \mathrm{~m} \mathrm{~s}^{-1}$ and $\overline{\alpha_{f}}=4.8^{\circ}$. During the present experiments, comparable pulse magnitudes up to $4 \mathrm{MPa}$ were observed.

In the present experiments, two different types of pressure pulses were identified and can be illustrated by figure 13. The pulses occurring before the $0.04 \mathrm{~s}$ mark are randomly distributed in time and space and are not repeated from cycle to cycle. These will be referred to as local pulses. On the other hand, the pulses located between 0.04 and $0.05 \mathrm{~s}$ occur virtually simultaneously, are of higher amplitude and are repeated each cycle. These will be referred to as global pulses; they produced substantial far-field noise.

\section{Cavitation structures associated with the pressure pulses}

The distinction between the two types of pressure pulse can be further elucidated by examining the high-speed movies. By synchronizing the high-speed movies and the transducer data acquisition, it was possible to determine exactly which cavitation structures are responsible for the generation of the large-amplitude pressure pulses.

Two different types of structures produced local pulses when they passed over the face of a foil surface transducer. The first type of flow structure (illustrated in photographs $a-c$ of figure 14) is a crescent-shaped region of low void fraction. These crescent-shaped regions appear randomly in the bubbly mixture that remains after the passage of the re-entrant jet. A close look at photograph (c) shows how complicated these flow structures can be since this crescent-shaped region appears to have some internal structure. Photographs $(b)$ and $(c)$ also show that more than one crescentshaped structure can be present at any moment in time. Some previous authors, most notably Shen \& Peterson (1978), Bark (1985), and McKenney \& Brennen (1994) have made passing reference to the existence of such perturbations within the cloud cavitation. Shen \& Peterson noted 'depressions in the cavity surface' while Bark refers to the 'deep inward buckling of the cavity interface'. 


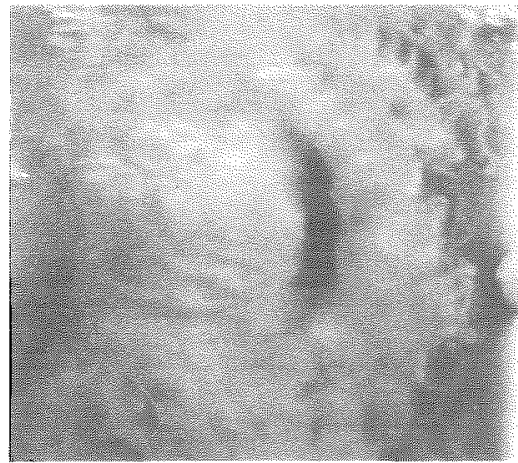

(a)

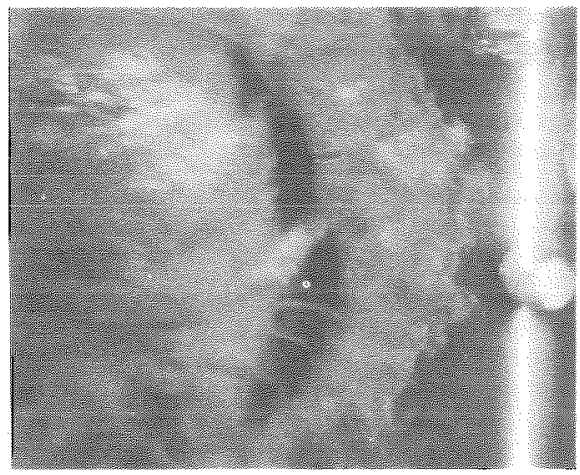

(c)

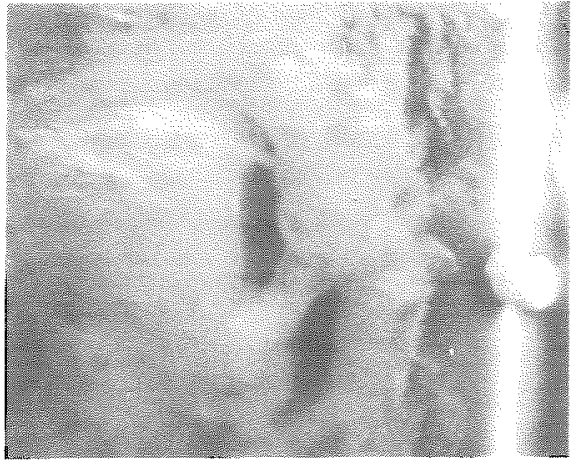

(b)

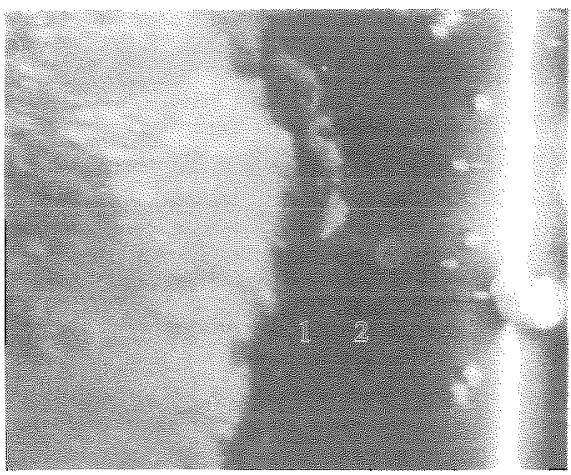

$(d)$

FIGURE 14. Local pulse structures in the cavitation on the suction surface of the NACA 0021 foil at $\sigma=0.9, k=0.7 \mathrm{~L}, U=8.5 \mathrm{~m} \mathrm{~s}^{-1}$ and $\overline{\alpha_{f}}=7^{3}$. The flow is from right to left; the field of view includes the four transducer locations and the leading edge is just at the right border. Crescent-shaped structures are seen in $(a),(b)$, and $(c)$ and a leading-edge event with two collapses (indicated by the arrows labelled 1 and 2 ) is seen in $(d)$.

An cxamination of the high-speed movies synchronized with acoustic data for over 40 oscillation cycles indicated the presence of a local pulse when the well-defined downstream boundary of such a low void fraction structure passed over a transducer; the pulse is thus registered when transitioning from a region of high void fraction to low void fraction. The downstream boundary of these low-void-fraction regions is observed to propagate into the bubbly mixture as the region expands. For example, the first local pulses registered by transducers \# 1 and \#3 in figure 13 were caused. by the passage of the downstream boundary of one of these crescent-shaped regions of low void fraction.

In addition, the movie and pressure data consistently displayed a local pulse when the upstream boundary, or leading edge, of the detached bubbly mixture passed over a transducer. This second type of local flow structure which also produces a local pulse is illustrated in photograph $(d)$ of figure 14. These 'leading-edge structures' are created when the sheet cavity detaches from the foil and they propagate downstream faster than the mixture velocity. Consequently, like the first kind of local pulse, this second type is associated with a transition from a region of high void fraction to a region of low void fraction. The structure of these leading-edge pulses often involves multiple pulses caused by several collapses and rebounds. This is exemplified in photograph 


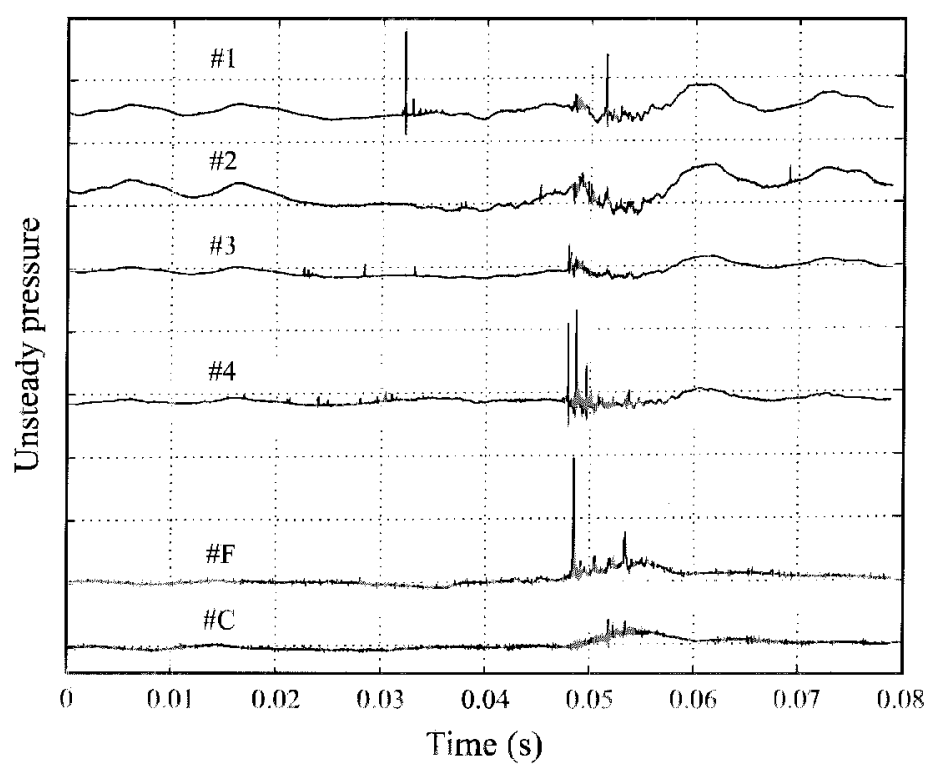

Figure 15. Transducer signals corresponding to the single oscillation cycle depicted in the photographs of figure 11. The vertical scale is $1 \mathrm{MPa} /$ div. for the foil surface transducers and $100 \mathrm{kPa} / \mathrm{div}$. for the floor and ceiling transducers. Data fot $\sigma=0.95, k=0.71, U=8.5 \mathrm{~m} \mathrm{~s}^{-1}$ and $\overline{\alpha_{f}}=7^{\circ}$.

$(d)$ of figure 14 in which the locations of the two collapse fronts are indicated by the arrows.

Another example of a leading-edge pulse is that detected by transducer \#1 just after the $0.03 \mathrm{~s}$ mark in figure 15; this occurs just before photograph $(e)$ of figure 11 . The distinct upstream boundary of the bubbly mixture passed over transducer \#1 just before this frame was taken. The second local pulse registered by transducer \#1 in figure 13 was also caused by the passage of a leading-edge structure.

It should be noted that a local pulse was not necessarily evident in the signal output every time either a crescent-shaped region or a leading-edge structure passed over a transducer. For example, the leading-edge structure evident in figure 11 generates a very large local pulse when it passes over transducer \#1 but does not produce a significant pulse when it later passes over transducer \#2. The absence of a significant local pulse was, however, the exception rather than the rule. On the other hand, the presence of a significant local pulse could almost always be traced to the presence of one of these local structures in the high-speed movies.

We now switch attention to the global pulses whose cause is also evident in the high-speed motion pictures. Figure 16 depicts four consecutive frames from one such movie and figure 17 contains the corresponding transducer output. The cavitation cloud which is the remnant of the attached sheet cavity undergoes a rapid and coherent collapse between frames $(b)$ and $(c)$ of figure 16. These frames were taken only $2 \mathrm{~ms}$ apart and lie somewhere in the region marked by the vertical lines in figure 17. The collapse of this cloud radiates a pressure pulse which is detected by all the transducers. The delay in the occurrence of the global pulse in the output of transducers \#1 and \#2 could be caused by the presence of travelling bubble cavitation near the leading edge of the foil which may cause a decrease in the local sonic speed. 


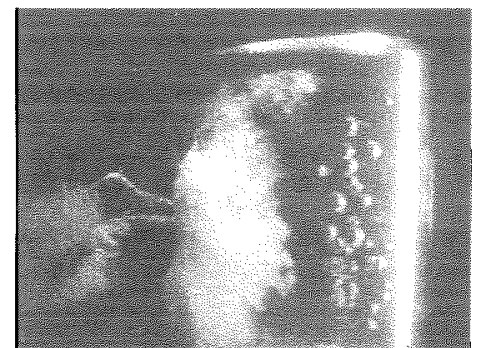

(a)

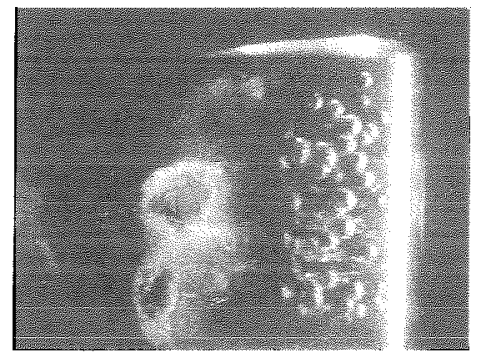

(c)

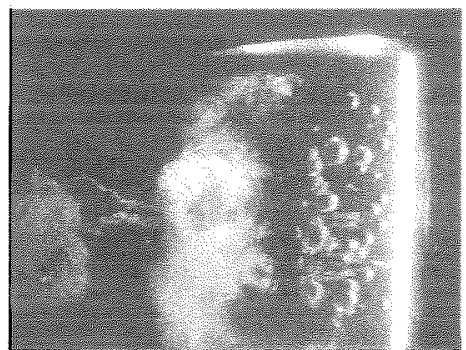

(b)

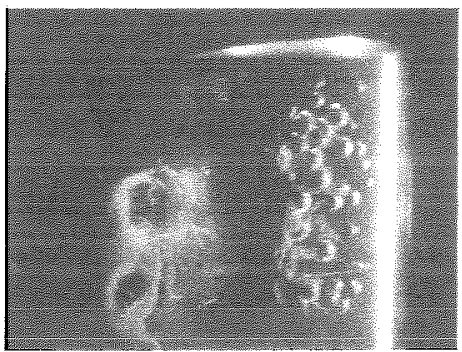

(d)

FIGURE 16. Consecutive high-speed movie frames ( $2 \mathrm{~ms}$ apart) showing global cloud collapse. These lie within the time interval marked in figure 17. The flow parameters are $\sigma=0.95, k=0.71$, $U=8.5 \mathrm{~m} \mathrm{~s}^{-1}$ and $\overline{\alpha_{f}}=7^{\circ}$.

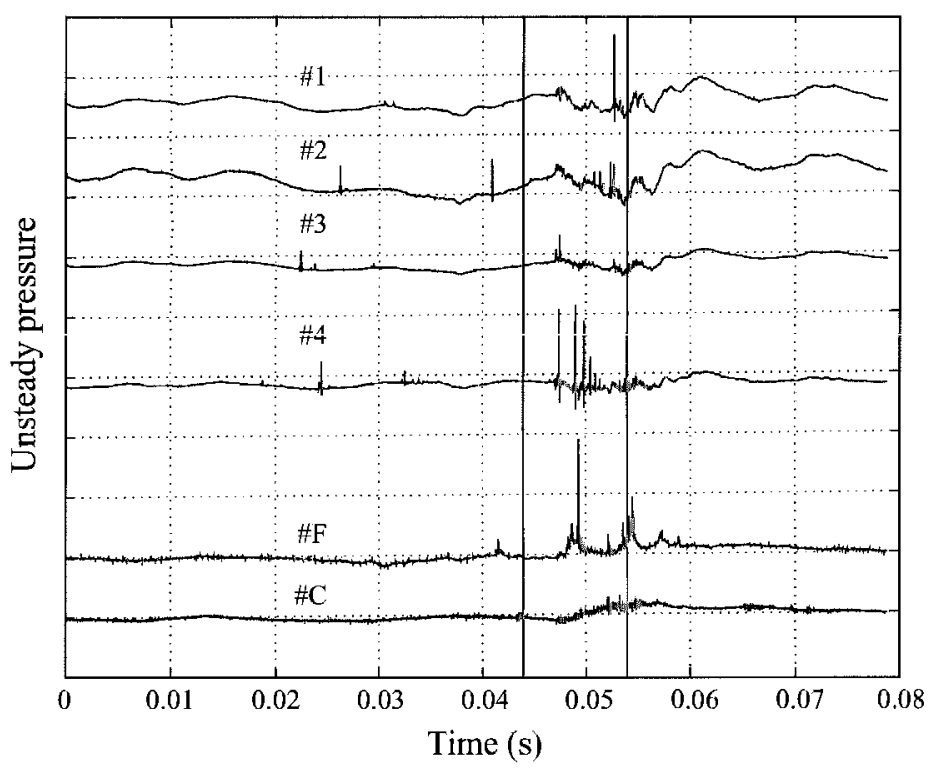

FIGURE 17. Transducer output corresponding to the global collapse photographs of figure 16 . The vertical scale is $1 \mathrm{MPa} /$ div. for the foil surface transducers and $100 \mathrm{kPa} /$ div. for the floor and ceiling transducers. Data for $\sigma=0.95, k=0.71, U=8.5 \mathrm{~m} \mathrm{~s}^{-1}$ and $\overline{\alpha_{f}}=7^{\circ}$. 


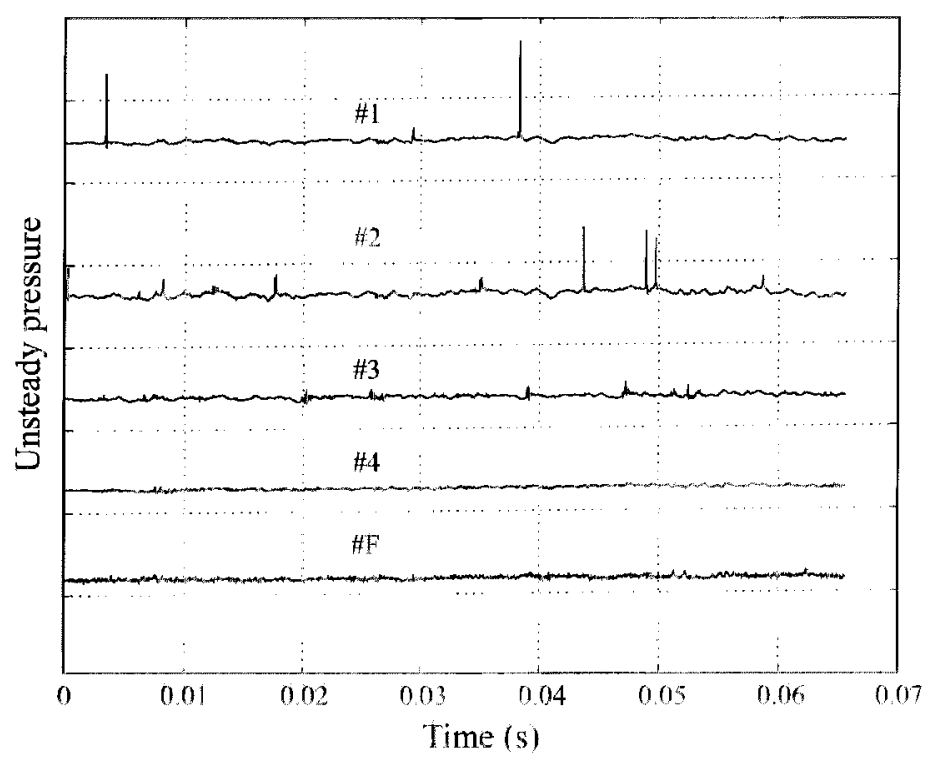

FIGURE 18. Typical transducer output during the stationary foil experiments. The vertical scale is $500 \mathrm{kPa} /$ div. for the foil surface transducers and $50 \mathrm{kPa} /$ div. for the floor transducer. Data for $\alpha_{f}=5^{\circ}, \sigma=0.95, U=8.5 \mathrm{~m} \mathrm{~s}^{-1}$.

The large pulses detected by the foil surface transducers and transducer \#F just before the $0.05 \mathrm{~s}$ mark in figure 15 were similarly identified as being caused by a global collapse. Yet another example is seen just before the $0.05 \mathrm{~s}$ mark in figure 13. This pulse, which was detected virtually simultaneously by all five transducers, was caused by a large coherent cloud collapse very similar to that depicted in figure 16.

It should be noted that none of these global cloud collapses involve large changes in the overall dimensions of the cloud; rather, collapse involves large changes in the void fraction distribution within the cloud. Rapid and non-uniform changes in the void fraction occur throughout the cloud interior as illustrated in figure 16. Also, the global collapse often generates multiple pulses, as exemplified in the output from transducers \#1, \#4 and \#F in figure 13, in the output of transducer \#4 in figure 15 and in the output from transducer \#4 in figure 17.

\section{Stationary foil experiments}

A typical set of output signals from the transducers during the experiments with a stationary or non-oscillating foil is shown in figure 18. The observed pulses are considerably smaller than with foil oscillation: the pulse magnitudes were typically only a few bars. There was also a substantial reduction in the radiated noise, and only local events were observed. The shedding of bubble clouds from the closure region of the attached cavity was a quite incoherent process, not characterized by any particular frequency. Rather, the clouds were detached in a continuous process which, therefore, did not produce distinct global events.

It should, however, be emphasized that these stationary foil experiments were carried out under conditions which produce relatively steady cavity flows. Specifically, the mean cavity length was short compared with the lengths which produce partial cavitation oscillation (Wade \& Acosta 1966; Brennen 1995). More-coherent global 
events and more-severe noise production occur when stationary foil experiments are performed with mean cavity lengths which produce partial cavitation oscillation. Note that the experiments of Kawanami et al. (1996) are of the latter type.

High-speed motion pictures taken at the same time as the data of figure 18 showed that while the cavitation structure is smaller and less coherent than observed on the oscillating foil, local events are still discernible in the cavitation on the foil surface. Also, each of the local pulses could be associated with the passage of either a crescent-shaped region or a leading-edge structure over the corresponding transducer. For example, the two local pulses between the 0.035 and $0.045 \mathrm{~s}$ marks in the output from transducers \#1 and \#2 in figure 18 were caused by the passage of the downstream boundary of a crescent-shaped region.

\section{Comparison of observed structures on two hydrofoils}

Although foil surface pressures were not measured with the much thinner NACA $64 \mathrm{~A} 309$ foil, it is valuable to compare the observed cloud cavitation structures observed in the high-speed motion pictures of this foil with those described above for the NACA 0021 hydrofoil. First, both types of local event structures are clearly evident with the thinner foil and the dynamics of both the crescent-shaped regions and the leading-edge structures do not appear to differ significantly from those seen on the NACA 0021 foil. Global events are also present and the global cloud collapse behaves as described above. Indeed, the only significant difference in the stages of cloud cavitation observed on the two foils is the presence of travelling bubble cavitation on the NACA 0021 foil for a longer fraction of the foil oscillation period and this difference is of little consequence for the present discussion.

The common appearance of the local and global pulse events on two foils with substantially different cross-sections and surface pressure distributions suggests that these structures may be common to a wide range of cloud cavitation flows.

\section{Parametric variations in the impulse magnitude}

The experimental results depicted in figures 19-21 illustrate the variation of the average acoustic impulses with reduced frequency, cavitation number, mean angle of attack and tunnel velocity for both the stationary and the oscillating foil In each figure the calculated impulses are presented for both the global and local pressure pulses detected by the four foil surface transducers and transducer \# $\mathrm{F}$. The average acoustic impulse is defined as the total impulse of all events divided by the number of foil oscillation cycles (usually 40).

Figure 19(a) illustrates the variation in the magnitudes of the local and global impulses with reduced frequency at a constant cavitation number, $\sigma$, and tunnel velocity, $U$. The conditions of this figure correspond to nominally steady partial cavitation on the stationary foil and result in virtually no global pulses. With foil oscillation, there appear to be ranges of reduced frequency around unity within which coherent collapses produce large global pulses. For example, the reduced frequencies $k=0.76$ and $k=1.22$ seem to produce particularly large global impulses as shown in figure 19. This general pattern of variation with reduced frequency is similar to that seen in the radiated noise by McKenney \& Brennen (1994). We also note from figure 19 that the spatial distribution of the impulses is similar for all reduced frequencies: they tend to be larger at mid-chord where the sheet cavitation breaks up and collapses. 

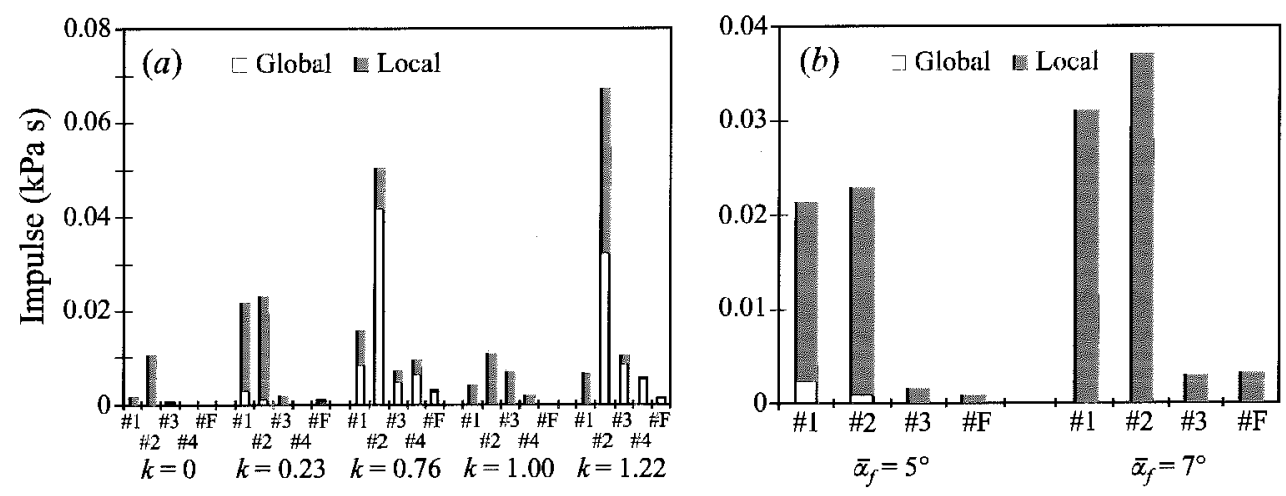

Figure 19. (a) The effect of reduced frequency, $k$, on the impulse, $I$, at $\overline{\alpha_{f}}=5^{\circ}$. (b) Impulse data for $k=0.23$ at two different mean angles of attack, $\overline{\alpha_{f}}=5^{\circ}$ and $7^{\circ}$. Data for $\sigma=0.95$ and $U=8 \mathrm{~m} \mathrm{~s}^{-1}$.
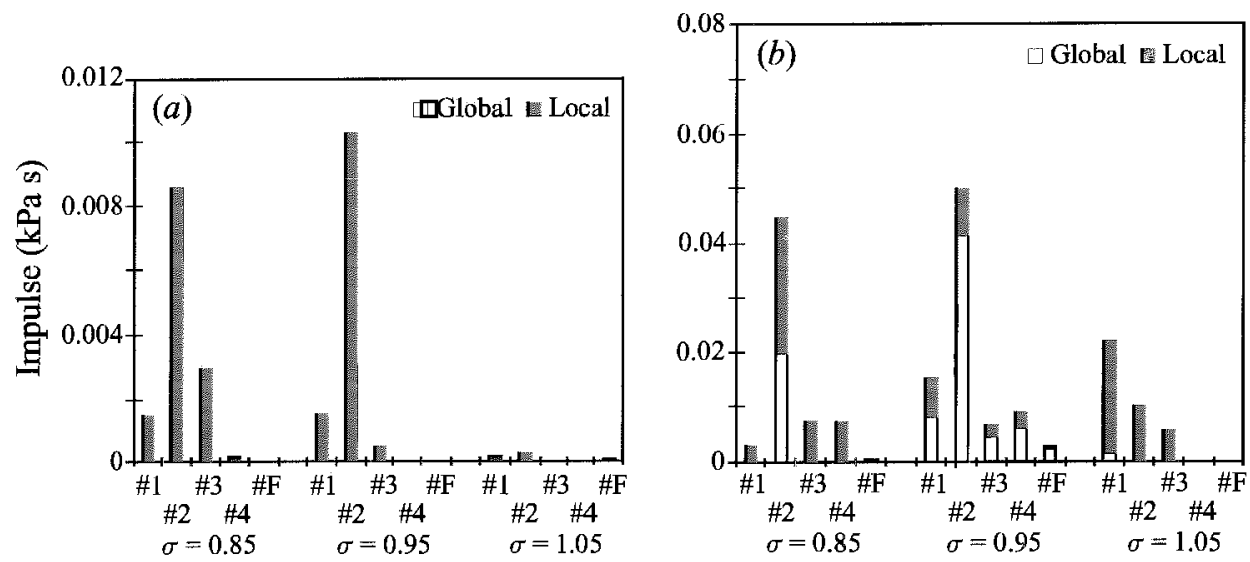

FIGURE 20. Effect of cavitation number, $\sigma$, on the impulse, $I$. (a) Data for the stationary foil at $\alpha_{f}=5^{\circ}$ and $U=8 \mathrm{~m} \mathrm{~s}^{-1}$. (b) Data for the oscillating foil with $k=0.76, U=8 \mathrm{~m} \mathrm{~s}^{-1}$ and $\overline{\alpha_{f}}=5^{\circ}$.

Figure 19(b) presents data for two mean angles of attack, $\overline{\alpha_{f}}$, with all other parameters rernaining the same. Note that the total impulse registered by every transducer increases when $\overline{\alpha_{f}}$ is increased from $5^{\circ}$ to $7^{\circ}$. Moreover, the spatial distribution remains almost unchanged. Curiously, however, global impulses are virtually absent at the larger angle of attack. This is another reflection of how the most-coherent dynamics are confined to quite small regions in the parameter space and yet large impulsive surface loadings occur within a much larger domain.

Figure 20 illustrates the variations in the impulses with the cavitation number, $\sigma$. With the stationary foil, the local impulses appear rather suddenly when $\sigma$ is reduced below unity. The largest impulses occur near transducer \#2, close to the cavity closure region; however, as $\sigma$ is decreased to 0.85 the values at \#3 increase as the cavitation collapse extends to that location. Le et al. (1993) also noted that the largest pulses on their stationary foil occurred near cavity closure. When the foil is oscillating, the global impulses produced are also very sensitive to changes in cavitation number with the largest global impulses occurring in the middle of the cavitation number range. A similar effect was detected in the radiated impulses by McKenney \& Brennen (1994) and Reisman et al. (1994), but the occurrence of cloud cavitation in the present 

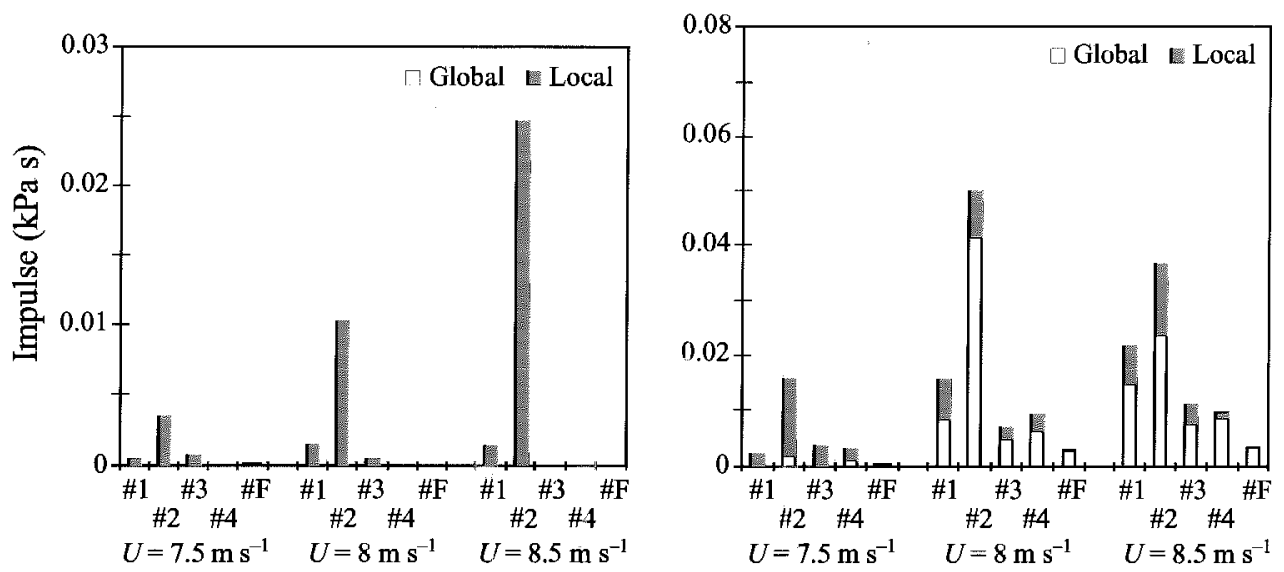

FIGURE 21. Effect of tunnel velocity, $U$, on the impulse, $I$. (a) Data for a fixed angle of attack of $5^{\circ}$ and $\sigma=0.95$. (b) Data for the oscillating foil with $0.72<k<0.81, \sigma=0.95$, and $\overline{\alpha_{f}}=5^{\circ}$.

experiments is more sensitive to changes in $\sigma$, perhaps due to the larger thickness of the NACA 0021 foil.

It is important to emphasize that extremely large local pressure pulses occur at cavitation numbers and flow conditions which do not produce global pulses or high levels of radiated noise. For example, an average local acoustic impulse in excess of $0.025 \mathrm{kPa} \mathrm{s}$ is observed on \#2 in the oscillating foil data of figure 20 at $\sigma=0.85$. Moreover, both with the stationary foil and with the oscillating foil (at high cavitation numbers), large local impulses occur on the foil surface without any substantial radiated impulse being registered by transducer \#F. These observations suggest that significant cloud cavitation damage could occur on a foil which is not exhibiting large acoustic radiation. Conversely, global pulses always produce substantial radiated noise.

The variations with tunnel velocity, $U$, are illustrated in figure 21 (since the frequency was held constant at $12.7 \mathrm{~Hz}$ in the oscillating case, there is also a small change in the reduced frequency as the tunnel velocity changes). With the stationary foil, the local impulses increase rapidly with increasing velocity as one would expect since cavitation noise normally scalcs with the velocity raised to some large power. Note again the predominance of events near transducer \#2. The total impulse also increases with increasing tunnel velocity when the foil oscillates, as shown figure 21 . This increase occurs at every measuring location with the single exception of the value measured by the \#2 transducer at $U=8.5 \mathrm{~m} \mathrm{~s}^{-1}$.

\section{Global pulse origin and propagation speed}

By examining the time delays in the global pulse signals from the five transducers (\#1-\#4 plus \#F) it was possible to extract some information on the location of the origin of the global pulses and on the speeds of propagation of those pulses through the inhomogeneous environment which exists near the surface of the hydrofoil at the time of the global cloud collapse.

For one representative flow condition $\left(\sigma=0.95, k=0.76, U=8 \mathrm{~m} \mathrm{~s}^{-1}, \overline{\alpha_{f}}=5^{\circ}\right)$ the transducer signals for 41 global pulse events were analysed. The location of the origin of the global pulses could be estimated by noting, for each event, the transducer which first registers the global pressure pulse. The number of events (out of 41) for which 


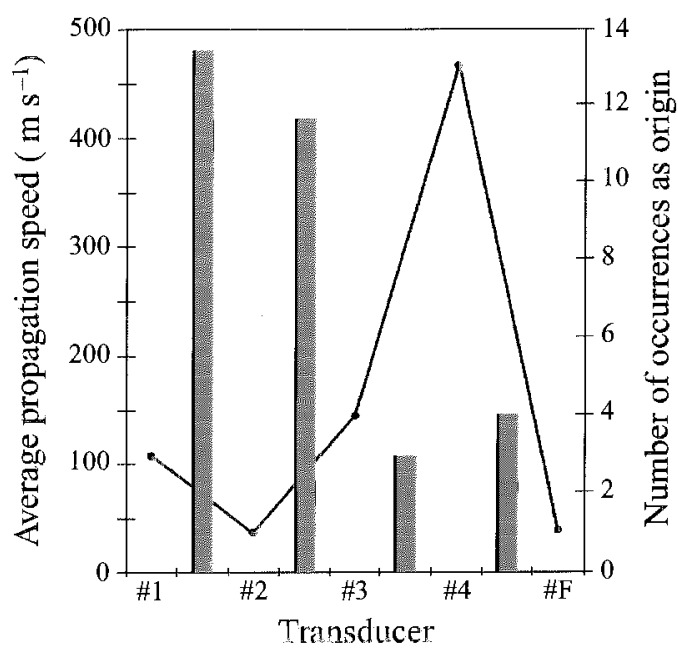

FIGURE 22. Speed of propagation (vertical bars) and location of the origin of the global pressure pulses (line). Data for $\sigma=0.95, k=0.76, U=8 \mathrm{~m} \mathrm{~s}^{-1}$, and $\overline{\alpha_{f}}=5^{\circ}$.

the origin could be so identified is shown in figure 22 . We note that global pulses most frequently originated near transducer \#4; this is consistent with the high-speed movie observations (e.g. figure 16) of global cloud collapse at this operating condition.

Furthermore, the speed of propagation of the global pressure pulse could be calculated from the time intervals separating the arrival of pulses at neighbouring transducers. These time intervals were converted to propagation speeds and are presented by the bar graphs in figure 22. In all cases, the propagation speeds are a small fraction of the sonic speed in either the pure liquid or pure vapour phase. The speeds for the rear intervals on the foil are substantially slower than the speeds for the forward intervals perhaps because, at the moment of cloud collapse, there is a sharp increase in the void fraction downstream of the \#3 transducer. This is due to the fact that the bubbly remains of the sheet cavity have been convected to this location at that moment in time.

But these speeds represent the rates at which the acoustic pulses are propagated away from the cloud. For reasons discussed later, it is also of interest to estimate the speed of propagation of the collapse process inside the cavitation cloud. The final stage of global cloud collapse proceeds very rapidly as was dernonstrated in frames $(b)$ and $(c)$ of figure 16 . The collapse process propagates through the cloud a distance of about $6 \mathrm{~cm}$, between frames $(b)$ and $(c)$ which are separated by $2 \mathrm{~ms}$. Thus a crude lower bound on the collapse propagation speed is $30 \mathrm{~m} \mathrm{~s}^{-1}$; the actual collapse could well travel much faster.

\section{Discussion}

The thesis of this paper is that the transducer signals and visual observations indicate that bubbly shock waves accompany the collapse of all or part of a cavitation cloud. When considered in conjunction with the early work of Mørch $(1980,1981$, 1982) and Hanson et al. (1981) and the numerical studies discussed in $\$ 2$, they support a new and somewhat unconventional view of the processes which occur in a cavitating flow. They indicate that the formation, focusing and propagation of bubbly shock waves play a critical role in generating the pressure pulses which lead to cavitation 
noise and damage. In the next two subsections, we examine the current observations of local and global cavitation shock structures in the context of previous investigations of bubbly shock waves.

First we examine more closely the evidence associated with the local pulse structures.

\subsection{Bubbly shock waves in local cavitation shock structures}

The large pressure pulse detected when a boundary of a local pulse structure passes over a transducer (along with the geometrically coherent and abrupt change in void fraction across the boundary) indicates that these structures consist of bubbly shock waves. Here we compare the characteristics of these structures with observations of bubbly shock waves in other contexts.

With both crescent-shaped regions and leading-edge structures, the boundary whose passage corresponds to the local pulses was observed to propagate into the region of high void fraction. The downstream boundary of the crescent-shaped region of photograph $(a)$ of figure 14 and the entire upstream boundary of the bubbly mixture depicted in photograph $(d)$ are both propagating into the region of larger void fraction since they are observed to be travelling downstream faster than the speed of the bubbly mixture. Thus they are bubbly shock waves propagating to the left in the photographs. Estimates from the movies of the propagation speed of these local pulses were quite scattered but appeared significantly larger than the flow velocities.

Noordzij \& van Wijngaarden (1974) conducted both experimental and analytical investigations of bubbly shock waves in the much simpler environment of a bubbly air/liquid mixture in a vertical tube. Their experimentally generated shocks were characterized by a change in void fraction across the shock greater than $80 \%$, a value which would not be inconsistent with the shock in the photographs of figure 14 .

Noordzij \& van Wijngaarden also examined the internal structure of their bubbly shocks which exhibit a series of oscillations after the passage of the leading shock front. Brennen (1995) presents calculations of similar shock structures for a cavitating flow and shows that the oscillations become a series of collapses (and rebounds) which produce a train of radiated acoustic pulses rather than a single pulse. The present experimental observations of local pulse structures clearly show that multiple pulses are frequently part of the structure of local pulse events. Not only is this apparent in the transducer traces (for example, transducer \#1 in figure 15) but can also be observed in the movies as indicated by the two arrows in photograph (d) of figure 14. Indeed, the presence of multiple peaks and several rebounds and collapses were commonly observed in leading-edge structures.

Another notable feature is the curvature of the crescent-shaped regions. Recognizing that these structures are propagating to the left in figure 14, a possible explanation for this curvature is that the void fraction is lower (and, therefore, if the void fraction is above about 0.5 , the shock speed is slower) near mid-span in the centre of the photograph. Indeed, large spanwise variations in the void fraction do result from the non-uniform forward penetration of the re-entrant jet. Recently, Kameda \& Matsumoto (1995) observed a similar curved structure for gas/liquid bubbly shocks in vertical tubes when the void fraction varied over the cross-section of the tube.

\subsection{Bubbly shock waves in global cavitation shock structures}

We now consider the global cloud collapse observations in the context of the spherical cloud calculations described in $\S 2$. Of course, the clouds in the present experiments (e.g. figure 16) are far from spherical. And, even in a homogeneous medium, gasdynamic shock focusing can be quite complex and involve significant nonlinear effects (see, 
for example, Sturtevant \& Kulkarny 1976). Nevertheless, it seems evident that once collapse is initiated on the surface of a cloud, the propagating shock will focus and produce large local pressure pulses and radiated acoustic pulses. It is not, however, clear exactly what form the foci might take in the highly non-uniform, threedimensional bubbly environment of a cavitation cloud on a hydrofoil. Nevertheless, the evidence we have presented here supports the thesis that shock wave propagation and focusing is an integral part of cloud dynamics and acoustics. For example, we note that in concert with the numerical results the observed global collapse process does not involve large changes in the overall dimensions of the cloud; rather it involves large changes in the void fraction distribution within the cloud ( $\$ 9)$. It was also noted during the experiments that the global collapse often generated multiple pulses in quick succession (e.g. figures 13, 15, 17 and 7) and these have a structure very similar to the radiated noise predicted by spherical cloud calculations. Also the evidence suggests that a certain cavitation number must be reached before global collapse occurs and this may be equivalent to the theoretical finding that the cloud interaction parameter, $\beta$, must exceed a critical value (of the order of unity) before major shock focusing occurs.

Quantitative comparisons are less easily made because of the lack of information on the appropriate values for the void fraction, $\alpha$, (and, therefore, for $\beta$ ) in the expeniments. Despite this difficulty, it is valuable to attempt some quantification; using guessed values of $\alpha_{0}=0.5 \%, A_{0}=5 \mathrm{~cm}$ and $R_{0}=500 \mu \mathrm{m}$ in the numerical model (as well as $\sigma=0.45$ and $C_{\text {mini }}=-0.75$ ) we calculate a maximum radiated acoustic pressure amplitude of $120 \mathrm{kPa}$ and a time interval between the multiple pulses of $0.7 \mathrm{~ms}$. These are within one order of magnitude of the experimental measurements for this condition, namely a maximum amplitude of $650 \mathrm{kPa}$ and a multiple pulse interval of $5 \mathrm{~ms}$.

\section{Concluding remarks}

Experimental measurements with hydrofoils experiencing cloud cavitation have shown that very large pressure pulses occur within the cloud and are radiated away from it during the collapse process. Within the cloud, these pulses can have magnitudes as large as 30 bar and durations of the order of $10^{-4} \mathrm{~s}$. These are certainly sufficient to explain the enhanced noise and cavitation damage associated with cloud cavitation. For example, the large impulsive surface loadings due to these pulses could be responsible for the foil darnage reported by W. B. Morgan (1995, personal communication), who observed propeller blade trailing edges bent away from the suction surface and toward the pressure surface.

Moreover, we have demonstrated that these pressure pulses are associated with several distinct cavitation structures which can be observed visually and which propagate through the bubbly mixture. We argue that these structures are different manifestations of the propagation of bubbly shock waves as a part of the internal mechanics of cloud collapse as had been previously anticipated by Mørch $(1980,1981,1982)$ and Hanson et al. (1981) and documented more recently by the calculations of spherical cloud collapse by Wang \& Brennen $(1994,1995)$.

Thus we suggest a new perspective on cavitation damage and noise in flows which involve large collections of cavitation bubbles with a sufficiently large void fraction (or, more specifically, a large enough $\beta$ ) so that the bubbles interact and collapse coherently. For a sufficiently large $\beta$, the calculations show that bubble collapse is initiated on the surface of the cloud and propagates into the cloud interior as a bubbly 
shock wave. Then geometric focusing produces the very high impulsive pressures and the radiated acoustic pulses which are characteristic of cloud cavitation.

Furthermore, the experiments reveal several specific shock wave mechanisms within the cavitation. One of these is the mechanism by which the large coherent collapse of a finite cloud of bubbles occurs; we have referred to these as global events and, as a result of the earlier computational work on spherical clouds, these findings were not wholly unexpected. A more unexpected result was the discovery of more localized bubbly shock waves propagating within the bubbly mixture in several forms, as crescent-shaped regions and as leading-edge structures. These seem to occur when the behaviour of the cloud is less coherent. They produce surface loadings which are within an order of magnitude of the more coherent events and could also be responsible for cavitation damage. However, because they are more localized, the radiated noise they produce is much smaller than that due to global events.

The phenomena described in this paper are expected to be important features in a wide range of cavitating flows since they were observed on two significantly different hydrofoils and since similar structures were noted in the work of earlier investigators such as Shen \& Peterson (1978) and Bark (1985). However, the analytical results clearly suggest that the phenomena may depend strongly on the cloud interaction parameter, $\beta$. If this is the case, some very important scaling effects may occur. It is relatively easy to envision a model in which $\beta$ is too smail for cloud effects to be important but that the prototype would have a much larger $\beta$ due to the larger cloud size, $A_{0}$ (while the void fractions and bubble sizes are comparable). Under these circumstances, the model would not manifest the large cloud cavitation effects which would occur in the prototype.

This research program was supported by the Office of Naval Research under grant number N00014-91-J-1295. The authors also greatly appreciate the advice and encouragement of Professor Allan Acosta and the help of Douglas Hart, Beth McKenney, Fabrizio d'Auria, Roberto Zenit, Tricia Waniewski, Don Kwak, Any Herr and Amir Alagheband.

\section{REFERENCES}

D'AGostino, L. \& Bpennen, C. E. 1983 On the acoustical dynamics of bubble clouds. In ASME Cavitation and Multiphase Flow Forum, pp. 72-75.

D’Agostino, L. \& ERennen, C. E. 1989 Linearized dynamics of spherical bubble clouds. J. Fluid Mech. 199, 155-176.

Arakeri, V. H. \& Shangumanathan, V. 1985 On the evidence for the effect of bubble interference on cavitation noise. J. Fluid Mech. 159, 131-150.

Arndt, R. E. A., Ellis, C. R. \& PAUL, S. 1993 Preliminary investigation of the use of air injection to mitigate cavitation erosion. In Proc. ASME Symp. on Bubble Noise and Cavitation Erosion in Fluid Systems. FED-176, pp. 105-116.

Bark, G. 1985 Developments of distortions in sheet cavitation on hydrofoils. In Proc. ASME Intl Symp. on Jets and Cavities, pp. 470-493.

BARK, G. \& Berlekom, W. B. vaN 1978 Experimental investigations of cavitation noise. In Proc. 12th ONR Symp. on Naval Hydrodynarnics, pp. 470493.

BlaKe, W. K. 1986 Mechanics of Flow-induced Sound and Vibration. Academic.

Blake, W. K., Wolpert, M. J. \& GeiB, F. E. 1977 Cavitation noise and inception as influenced by boundary-layer development on a hydrofoil. J. Fluid Mech. 80, 617-640.

Brennev, C. E. 1995 Cavitation and Bubble Dynamics. Oxford University Press.

Ceccio, S. L. \& Brennen, C. E. 1991 Observations of the dynamics and acoustics of travelling bubble cavitation. J. Fluid Mech. 233, 633-660. 
Chahine, G. L. 1982 Cloud cavitation: theory. In Proc. 14th ONR Symp. on Naval Hydrodynamics, pp. 165-194.

Chahine, G. L. \& Duraiswami, R. 1992 Dynamical interactions in a multibubble cloud. Trans. ASME: J. Fluids Engng 114, 680-686.

FitzPatrick, H. M. \& Strasberg, M. 1956 Hydrodynamic sources of sound. In Proc. First ONR Symp. on Naval Hydrodynamics, pp. 241-280.

FRANC, J. P. \& MicheL, J. M. 1988 Unsteady attached cavitation on an oscillating hydrofoil. J. Fluid Mech. 193, 171-189.

GATES, E. M. 1977 The influence of free stream turbulence, free stream nuclei populations, and a drag-reducing polymer on cavitation inception on two axisymmetric bodies. $\mathrm{PhD}$ thesis, Cal. Inst. of Tech.

Hanson, I., Kedrinskit, V. K. \& MørCh, K. A. 1981 On the dynamics of cavity clusters. J. Appl. Phys. 15, 1725-1734.

HaRT, D. P., BrenNen, C. E. \& Acosta, A. J. 1990 Observations of cavitation on a three dimensional oscillating hydrofoil. In ASME Cavitation and Multiphase Flow Forum. FED-98, pp. 49-52.

JAKOBSEN, J. K. 1964 On the mechanism of head breakdown in cavitating inducers. Trans. ASME: J. Basic Engng. 86, 291-304.

KAMEDA, M. \& MATsumoto, Y. 1995 Structure of shock waves in a liquid containing gas bubbles. In IUTAM Symp. on Waves in Liquid/Gas and Liquid/Vapour Two-Phase Systems, pp. 117-126.

Kawanami, Y., Kato, H., Yamaguchi, H., Tagaya, Y. \& Tanimura, M. 1996 Mechanism and control of cloud cavitation. In Proc. ASME Symp. on Cavitation and Gas-Liquid Flows in Fluid Machinery and Devices. FED-236, pp. 329-336.

KNAPP, R.T. 1955 Recent investigations of the mechanics of cavitation and cavitation damage Trans. $A S M E$ 77, 1045-1054.

Kubota, A., Kato, H. \& Yamaguchi, H. 1992 A new modelling of cavitating flows: a numerical study of unsteady cavitation on a hydrofoil section. J. Fluid Mech. 240, 59-96.

Kubota, A., Kato, H., Yamaguchi, H. \& MaEdA, M. 1989 Unsteady structure measurement of cloud cavitation on a foil section using conditional sampling. Trans. ASME: J. Fluids Engng 111, 204-210.

Kuhn de Chizelle, Y., Ceccio, S. L. \& Brennen, C. E. 1995 Observations, scaling and modeling of travelling bubble cavitation. J. Fluid Mech. 293, 99-126.

Kumar, S. \& Brennen, C. E. 1991 Non-linear effects in the dynamics of clouds of bubbles. J. Acoust. Soc. Am. 89, 707-714.

KuMAR, S. \& BRENNEN, C. E. 1992 Harmonic cascading in bubble clouds. In Proc. Intl Symp. on Propulsors and Cavitation, pp. 171-179.

Kumar, S. \& Brennen, C. E. 1993 a Some nonlinear interactive effects in bubbly cavitating clouds. J. Fluid Mech. 253, 565-591.

KumAR, S. \& BrenNen, C. E. $1993 b$ A study of pressure pulses generated by travelling bubble cavitation. J. Fluid Mech. 255, 541-564.

Lange, D. F. De, Bruin, G. J. de \& WijngaARden, L. van 1994 On the mechanism of cloud cavitation - experiment and modeling. In Proc. 2nd Intl Symp. on Cavitation, Tokyo, pp. 45-50.

Le, Q., Franc, J. M. \& Michel, J. M. 1993 Partial cavities: global behaviour and mean pressure distribution. Trans. ASME: J. Fluids Engng 115, 243-248.

LuSH, P. A. \& SKIPP, S. R. 1986 High speed cine observations of cavitating flow in a duct. Intl J. Heat Fluid Flow 7, 283-290.

McKenney, E. A. \& Brennen, C. E. 1994 On the dynamics and acoustics of cloud cavitation on an oscillating hydrofoil. In Proc. ASME Symp. on Cavitation and Gas-Liquid Flows in Fluid Machinery and Devices. FED-190, pp. 195-202.

MøRCH, K. A. 1980 On the collapse of cavity cluster in flow cavitation. In Cavitation and Inhomogenieties in Underwater Acoustics (ed. W. Lauterborn), pp. 95-100. Springer.

Mørсн, K. A. 1981 Cavity cluster dynamics and cavitation erosion. In Proc. ASME Cavitation and Polyphase Flow Forum, pp. 1-10.

MørCH, K. A. 1982 Energy considerations on the collapse of cavity cluster. Appl. Sci. Res. 38, 313.

NOORDZIJ, L. \& WIJNGAARDEN, L. vaN 1974 Relaxation effects, caused by relative motion, on shock waves in gas-bubble/liquid mixtures. J. Fluid Mech. 66, 115-143.

OмтA, R. 1987 Oscillations of a cloud of bubbles of small and not so small amplitude. $J$. Acoust. Soc. Am. 82, 1018-1033. 
Prosperetti, A. 1988 Bubble-related ambient noise in the ocean. J. Acoust. Soc. Am. 84, 1042-1054.

REISMAN, G. E. 1997 Dynamics, acoustics and control of cloud cavitation on hydrofoils. PhD thesis, Cal. Inst. of Tech.

Reisman, G. E. \& BRenNen, C. E. 1996 Pressure pulses generated by cloud cavitation. In ASME Symp. on Cavitation and Gas-Liquid Flows in Fluid Machinery and Devices. FED-236, pp. 319 328.

Reisman, G. E., McKenney, E. A. \& Brennen, C. E. 1994 Cloud cavitation on an oscillating hydrofoil. In Proc. 20th ONR Symp. on Naval Hydrodynamics, pp. 78-89.

SheN, Y. \& PeTERSON, F. B. 1978 Unsteady cavitation on an oscillating hydrofoil. In Proc. 12th ONR Symp. on Naval Hydrodynamics, pp. 362-384.

Shen, Y. \& Peterson, F. B. 1980 The influence of hydrofoil oscillation on boundary layer transition and cavitation noise. In Proc. 13th ONR Symp. on Naval Hydrodynamics, pp. 221-241.

SMEREkA, P. \& BANERJEe, S. 1988 The dynamics of periodically driven bubble clouds. Phys. Fluids 31, 3519-3531.

SoYama, H., KaTo, H. \& OBA, R. 1992 Cavitation observations of severely erosive vortex cavitation arising in a centrifugal pump. In Proc. Third IMechE Intl Conf. on Cavitation, pp. 103-110.

Sturtevant, B. \& KulkarnY, V. J. 1976 The focusing of weak shock waves. J. Fluid Mech. 73, $651-680$.

WADE, R. B. \& AcostA, A. J. 1966 Experimental observations on the flow past a plano-convex hydrofoil. Trans. ASME: J. Basic Engng 88, 273-283.

WANG, Y.-C. 1996 Shock waves in bubbly cavitating flows. PhD thesis, Cal. Inst. of Tech.

WANG, Y.-C. \& BRENNEN, C. E. 1994 Shock wave development in the collapse of a cloud of bubbles. In ASME Cavitation and Multiphase Flow Forum. FED-194, pp. 15-20.

WANG, Y.-C. \& BrenNen, C. E. 1995 The noise generated by the collapse of a cloud of cavitation bubbles. In ASME/JSME Symp. on Cavitation and Gas-Liquid Flow in Fluid Machinery and Devices. FED-226, pp. 17-29.

WIJNGAARDEN, L. VAN 1964 On the collective collapse of a large number of gas bubbles in water. In Proc. 11th Int. Conf. Appl. Mech. pp. 854-861. 